Western University

Scholarship@Western

Digitized Theses

Digitized Special Collections

1974

\title{
Tauberian Theorems On A Scale Of Abel-type Summability Methods
}

Bruce Brigham Watson

Follow this and additional works at: https://ir.lib.uwo.ca/digitizedtheses

\section{Recommended Citation}

Watson, Bruce Brigham, "Tauberian Theorems On A Scale Of Abel-type Summability Methods" (1974). Digitized Theses. 727.

https://ir.lib.uwo.ca/digitizedtheses/727

This Dissertation is brought to you for free and open access by the Digitized Special Collections at Scholarship@Western. It has been accepted for inclusion in Digitized Theses by an authorized administrator of Scholarship@Western. For more information, please contact tadam@uwo.ca,

wlswadmin@uwo.ca. 
A

TAUBERIAN THEOREMS ON A SCAYE OF ABEL-TYPE SUMMABILITY METHODS $\therefore$

by

Bruce Brigham watson

Department of Mathematics

$\checkmark$

Submitted in partial fulfillment of the requirements for the degree of Doctor af Philosophy

Faculty of Graduate studies

The University of Western Ontario

London, ontario

February, 1974

(C) Bruce Brigham Watson 1974. 
ACKNOWLEDGEMENTS

I would -like to express my sincere gratitude to Professor David Borwein for his kindness, encouragement arid academic guidance. I am also indebted to the National Research Council of Canada for the award of a National Research Council Scholarship, and the Government of. Ontario for the award of an Ontario Graduate Fellowship. Finally, I would like to thank Mrs. J. Stacey for the typing of the thesis. 
CERTIFICATE OF EXAMINATION ................ i i

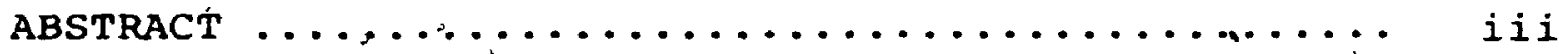

ACKNOWEDGEMENTS $\ldots \ldots \ldots \ldots \ldots \ldots \ldots \ldots \ldots \ldots \ldots \ldots \ldots$ iv

TABLE OF CONTENTS $\ldots \ldots \ldots \ldots \ldots \ldots \ldots \ldots \ldots \ldots$

CHAPTER $1 \ldots \ldots \ldots \ldots \ldots \ldots \ldots \ldots \ldots \ldots \ldots \ldots \ldots \ldots \ldots \ldots \ldots \ldots \ldots$

1.1 Introduction $\ldots \ldots \ldots \ldots \ldots \ldots \ldots \ldots \ldots \ldots \ldots \ldots \ldots \ldots$

1.2 Summability Methods .................. 2 -

1.3 Abel-Type Methods and the Logarithmic

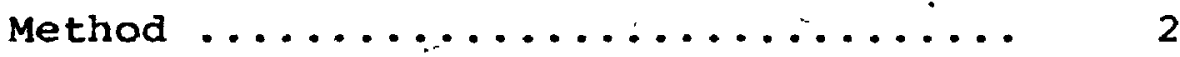

1.4, Regularity and Inclusion Theorems for the

$\left(A_{\lambda}\right)$ and $(L)$ Methods $\ldots \ldots . . . \ldots \ldots \ldots . . . .4$

T.5. Additional summability Methods. Cesaro ?

summability $(c, \alpha) \ldots \ldots \ldots \ldots \ldots \ldots \ldots$

1.6 Remarkg Concerning Theorems 1.2 and $1.4=\ldots$

CHAPTER 2 - A TAUBERIAN THEQREM .............. 12

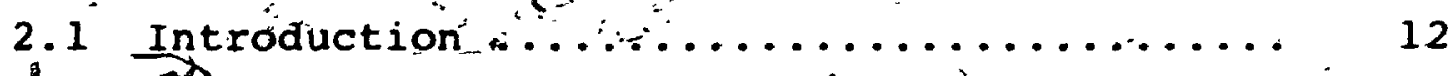

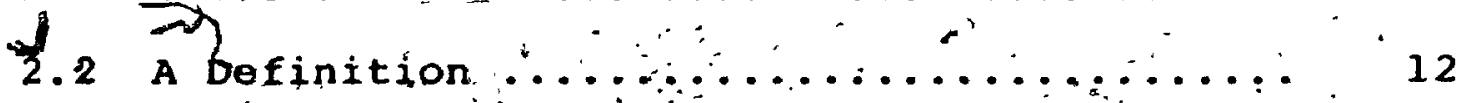

2.3 statément of the Theorem $\ldots \ldots \ldots . \ldots \ldots \ldots \ldots . . \ldots .12$

2.4 Some Lemmas $\ldots \ldots, \ldots \ldots \ldots \ldots \ldots \ldots \ldots \ldots \ldots \ldots \ldots \ldots \ldots \ldots \ldots \ldots$

2.5 Proof of Theorem $2.1 \ldots \ldots \ldots \ldots \ldots \ldots \ldots \ldots \ldots$

2.6 comment ........................ 17 
CHAPTER 3 - A TAUBERIAN THEOREM OF SLOWLY

DECREASING TYPE $-1 \ldots \ldots \ldots \ldots . \ldots . \ldots$

3.1 Statement of the Theorem ............... 18

3.2 A Vijayaraghavan-Type Result ............ 19

- 3.3 Sone Preliminary Lemmas ............... 20

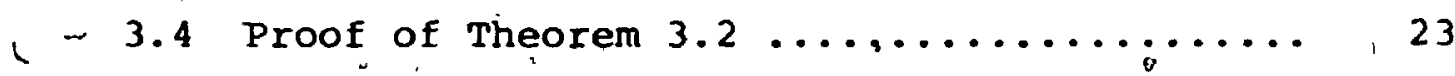

3.5 A Tauberiän Theorem of wiener.......... 29

3.6 Proof of Theorem. $3.1 \ldots \ldots \ldots \ldots \ldots \ldots \ldots \ldots . \ldots . \ldots 29$

3.7 A Tauberian Theorem-of Q-Type ........... 31

CHAPTER 4 - A TÁUBERIAN THEOREM OF SLOWLY. DECREASING TYPE ${ }^{\star}$ - II ................ 35

4.1 Introduction $\ldots \ldots \ldots \ldots \ldots \ldots \ldots \ldots \ldots \ldots \ldots \ldots$

4.2 Some Lemmas $\ldots \ldots \ldots \ldots \ldots \ldots \ldots \ldots \ldots \ldots . \ldots \ldots$

4.3 A Counterexample .................. 37

4.4. A Tauberian Theorem for (L). to $\left(\mathrm{A}_{\lambda}\right)$

Summability .................. 38

4.5 Methods of Summability Based on Power

Series .................. 38

4.6 Some preliminary temmas ............... 41

4.7 Proof of Theorem $4.2 \ldots \ldots \ldots \ldots \ldots \ldots \ldots \ldots \ldots \ldots . \ldots 1$

4.8 A Condition on $\left\{n a_{n}\right\}$ which Implies

Relation $(4.4 .1) \ldots \ldots . \ldots \ldots \ldots \ldots . . . .$.

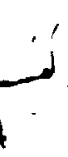


c

CHAPTER 5 - A TAUBERIAN THEOREM FOR ABELIAN SUMMABILITY. METHODS

54

5.1 Introduction

54

5.2 A Preliminary Lemma

54

5.3 Proof of Theorem 5.1

55

REFERENCES

57

VITA

61 
The author of this thesis has granted The University of Western Ontario a non-exclusive license to reproduce and distribute copies of this thesis to users of Western Libraries. Copyright remains with the author.

Electronic theses and dissertations available in The University of Western Ontario's institutional repository (Scholarship@Western) are solely for the purpose of private study and research. They may not be copied or reproduced, except as permitted by copyright laws, without written authority of the copyright owner. Any commercial use or publication is strictly prohibited.

The original copyright license attesting to these terms and signed by the author of this thesis may be found in the original print version of the thesis, held by Western Libraries.

The thesis approval page signed by the examining committee may also be found in the original print version of the thesis held in Western Libraries.

Please contact Western Libraries for further information:

E-mail: libadmin@uwo.ca

Telephone: (519) 661-2111 Ext. 84796

Web site: http://www.lib.uwo.ca/ 


\section{CHAPTER 1}

\subsection{INTRODUCMIOAं}

Let $\sum_{n=0}^{\infty} a_{n}$ be a series of real numbers and let $\left\{s_{n}\right\}$ denòte lits associated sequence of partial sums. That is.

$$
\begin{aligned}
\dot{s}_{n} & =a_{0}+a_{1}+\cdots+a_{n} n \geq 0 \\
s_{n} & =0
\end{aligned}
$$

The symbol $M$ is used throughout the theșis to denote a positive constant, independent of the variables under consideration, but not necessarily having the same. (2) value, at each occurrence.

We adopt the following familn conventions:

$$
\begin{aligned}
& f=D(g) \text { means }|f|<M g \\
& f=O(g) \text { means } f / g \rightarrow 0 \\
& f \sim g \text { means } f / g \rightarrow 1 .
\end{aligned}
$$

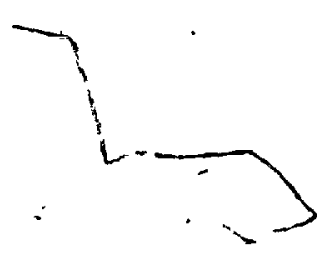

The theorems and lemmas in the thesis are numbered . chapterwise, and independently-x That is, Theorem 3.1 is the first theorem in Chapter Three, however, there may also be a Lemma 3.1. Relations are numbered according to the chapter and section in which they occur. For example, (3.2.4) is the fourth relation in section two of Chapter Three: 


\subsection{SUMMABILITY METHODS}

A summability method is a process $\mathrm{p}$ which assigns a.sum s to the series $\sum_{n=0}^{\infty} a_{n}$. When $s$ is finite, we say that $\sum_{n=0}^{\infty} a_{n}$ is p-summable to the sum s, and write $\sum_{n=0}^{\infty} a_{n}^{-}=s(P)$.

We shall also say that the sequence $\left\{s_{n}\right\}$ is P-convergent to the iimit.s, and write

$$
s_{n} \rightarrow s(P)
$$

A summability method $P$ is said to be rezuiar if $\mathbf{a}_{n}{ }^{\prime} \rightarrow \mathbf{s}(P)$ whenever the sequence $\left\{s_{n}\right\}$ converges to $s^{\prime}$ in the ordinary sense.

Given two summability methods $P$ and $Q, " P$ is said. to include $\mathrm{Q}$ if every sequence convergent by the method $Q$ is also convergent by the method $P$ to the same limit. This inclusion relationship will be written

$$
\therefore Q \subseteq P \text {. }
$$

If $P$ includes $Q$, and $Q$ includes $P$, then we say that $P$ is equivalent to $Q$ and write

$$
P \simeq Q
$$

1.3. ABEL-TYPE METHODSऽAND THE LOGARITHMIC METHOD Let $\lambda$, be any real number, and $\dot{\varepsilon}_{0}^{\lambda}=1$. Also set 


$$
\begin{aligned}
& \varepsilon_{n}^{\lambda}=\left(\begin{array}{c}
n+\lambda \\
n
\end{array}\right)=\frac{(\lambda+1)(\lambda+2) \ldots(\lambda+n)}{n !} \quad, \quad n=1,2, \ldots \\
& \sigma_{\lambda}(y)=(1+y)^{-\lambda-1} \cdot \sum_{n=0}^{\infty} \varepsilon_{n}^{\lambda} s_{n}\left(\frac{y}{1+y}\right)^{n} \quad y>\sigma \\
& L(\dot{y})=\frac{1}{\log (1+y)} \int_{n=9}\left(\frac{y}{n+1}\right)^{n+1}, \quad y>0 .
\end{aligned}
$$

The Abel-type methods $\left(A_{\lambda}\right)$ were introduced and studied by Borwein, $[2]$. The summability method $\left(A_{0}\right)$ is the ordinary Abel: method (A):

Evidently, we have the following:

(i) $s_{n}+s\left(A_{\lambda}\right)$ if and only if the expression

$$
\text { . }(1-x)^{\lambda+1} \sum_{n=0}^{\infty} \varepsilon_{n}^{\lambda} s_{n} x^{n}
$$

converges for all $x$ in the open interval $(0,1)$ and tends to $s$ as $x \rightarrow 1^{-}$.

$$
\begin{array}{rl}
(i i), s_{n} & s(L) \text { if and only if the expression } \\
& -\frac{1}{\log (1-x)} \sum_{n=0}^{\infty} \frac{s_{n}}{n+1} x^{n+1}
\end{array}
$$

converges for all $x$ in the open interval $(0,1)$ and tends to 8 as $x \rightarrow 1^{-}$. 


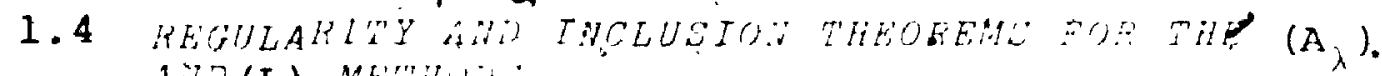
Aitz(L) ME'Litili.

In this section the regularity of the $\left(A_{\lambda}\right)$ and

summability methods is"discussed. Also, the scale of Abel-type methods is developed and the natural inclusion" between the $\left(A_{\lambda}\right)$ and $(L)^{\circ}$ summability mèthods is stated. For the sake of completeness, praofs of some of these known results are given.

THEOREM ' 1 : 1

The $\left(A_{\lambda}\right)$ methud is reguiar $\therefore r \lambda_{>}>-b$.

THEOREM ${ }^{\circ} 1.2$

$$
A_{\lambda+\varepsilon} \subseteq{ }_{\lambda}^{A} \text { for } \lambda>-1, \varepsilon>0 \text {. }
$$

THEOREM 1.3

$$
\text { The (L) method is realar. }
$$

THEOREM 1.4 :

$$
A_{\lambda} \subseteq \mathrm{L} \operatorname{for} \lambda>-1 \text {. }
$$

Theorems 1.1 and 1.3 are deduced from the following theorem on positive', regular methods of summability in $\left[7^{\circ}\right]$. THEOREM 1.5

Suppose, för $\mathrm{n}=0,1,2, \ldots$

\section{8}

$$
\begin{aligned}
& c_{n}(y) \geq 0 \quad \forall \quad y \$ 0 \\
& c_{n}(y) \rightarrow 0 \quad \therefore, y+\infty
\end{aligned}
$$

and.

$$
\sum_{n=0}^{\infty} c_{n}(y)=i \quad y>0
$$

$$
\text { If, for } \mathrm{Y}>\text { ? }
$$

$$
T(\dot{y})=\sum_{n=0}^{\infty} c_{n}(y) B_{n}
$$


then $\mathrm{T}(\mathrm{y}) \rightarrow \mathrm{s} \dot{a} \mathrm{~s}_{\mathrm{m}} \mathrm{Y} \rightarrow \infty \stackrel{w}{\mathrm{w} h e n e v e r \mathrm{~s}_{\mathrm{n}}} \rightarrow \mathrm{s}$

$(-\infty \leq s \leq \infty)$

The $T(y)$ of the above theorem becomes $\sigma_{\lambda}(y)$ and

$L(y)$ according as

$$
\begin{aligned}
c_{n}(y) & =(1+y)^{-\lambda-1} \varepsilon_{n}^{\lambda}\left(\frac{y}{1+y}\right)^{n} 0 \\
c_{n}(y) & =\frac{1}{(n+1) \log (1+y)}\left(\frac{y}{1+y}\right)^{n+1} .
\end{aligned}
$$

The proof of Theorem 1.2 requires some preliminary

lemmas.

LEMMA 1.1

If $\lambda>-1, \mu>-1$, and $|x|<1$, then

(i) $\sum_{n=0}^{\infty} \varepsilon_{n}^{\lambda} s_{n} x^{n}$ is. absolutely convèrgent if and only if

$\sum_{n=0}^{\infty} \varepsilon_{n}^{\mu} s_{n} \mathbf{x}^{n}$ is iasoluterly convergent.

(ii) $\sum_{n=0}^{\infty} \varepsilon_{n}^{\lambda} s_{n} x^{n}$ is absoluteiy convergent if and only if

$\sum_{\mathbf{n}=0}^{\infty} \frac{\mathbf{s}_{\mathbf{n}}}{\mathrm{n}+1} \mathrm{x}^{\mathbf{n}^{\prime}}$ is absolutely convergent.

PRQOF

(i) $\varepsilon_{n}^{\lambda} \sim \frac{n^{\lambda}}{\Gamma(\lambda+1)}$ as $n \rightarrow \infty$.

Thefefore

$\because n \rightarrow \infty$

$$
\lim _{n \rightarrow \infty}\left(\varepsilon_{n}^{\lambda}\right)^{1 / n}=1
$$

Hence the radius of convergence of $\sum_{n=0}^{\infty} \varepsilon_{n}^{\lambda} n^{n} x^{n}{ }^{n} \cdot$ the same 
as that of $\sum_{n=0}^{\infty} s_{n} x^{n}$. Therefore (i) follows.

6.9

(ii) $\lim _{n \rightarrow \infty}\left(\frac{1}{n+1}\right)_{0}^{1 / n}=1$.

Hence, as in (i), $\sum_{n=0}^{\infty} \frac{s_{n}}{n+1} x^{n}$ has the same radius of convergence as $\sum_{n=0}^{\infty} s_{n} x^{n}$ and the lemma follows.

$\because$ LEMMA 1.2

$$
\begin{aligned}
& \text { For } \lambda>-1, y>0, \varepsilon>0 \text {, ariz } n=0,1,2, \ldots \text { we have } \\
& \text { (1.4.1) } \frac{\Gamma(\lambda+\varepsilon+1)}{\Gamma(\lambda+1) \Gamma(\varepsilon)} \varepsilon_{n}^{\lambda+\varepsilon} y^{-\lambda-\varepsilon} \int_{0}^{y}(y-t)^{\varepsilon-1} t^{\lambda+n}(1+t)^{-\lambda-\varepsilon-1-n} d t \\
& \quad=\varepsilon_{n}^{\lambda}(1+y)^{-\lambda-1}\left(\frac{y}{1+y}\right)^{n}
\end{aligned}
$$

PROOF

$$
\text { Set } u=t / 1+t, x=y / 1+y \text { in the integral in }(1,4.1) \text {. }
$$

to obtain

$$
\begin{aligned}
& \int_{0}^{y}(y-t)^{\varepsilon-1} t^{\lambda+n}(1+t)^{-\lambda-\varepsilon-1-n} d t \\
= & (1-x)^{1-\varepsilon} \int_{-0}^{\dot{x}}(x-u)^{\varepsilon-1} u^{\lambda+n} d u \\
= & x^{\lambda+\varepsilon+n}(1-x)^{1-\varepsilon} \int_{0}^{1} t^{\varepsilon-1}(1-t)^{\lambda+n} d t \\
0= & \frac{y^{\lambda+\varepsilon+n} \Gamma(\varepsilon) \Gamma(\lambda+n+1)}{(1+y)^{\lambda+1+n} \Gamma(\lambda+\varepsilon+n+1)}
\end{aligned}
$$


Therefore

$$
\begin{aligned}
& \frac{\Gamma(\lambda+\varepsilon+1)}{\Gamma(\lambda+1) \Gamma(\varepsilon)} \varepsilon_{n}^{\lambda+\varepsilon} y^{-\lambda-\varepsilon} \cdot \int_{0}^{y}(y-t)^{\varepsilon-\frac{1}{2}} t^{\lambda+n}(1+t)^{-\lambda-\varepsilon-1-\dot{n}} d t \\
& =\varepsilon_{n}^{\lambda+\varepsilon} \frac{\Gamma(\lambda+1+n)}{\Gamma(\lambda+1)} \frac{\Gamma(\lambda+\varepsilon+1)^{2}}{\Gamma(\lambda+\varepsilon+1+n)}(\dot{1}+y)^{-\lambda-1}\left(\frac{y}{1+y}\right)^{n} \\
& =\varepsilon_{n}^{\lambda}(1+y)^{2 \lambda-1}\left(\frac{y}{1+y}\right)^{n} .
\end{aligned}
$$

This completes the proof of the lemma.

LRMMAA 1.3

$$
\therefore \quad \text { If } \lambda>-1, \varepsilon>0, y>0 \text { and } \sum_{n=0}^{\infty} \varepsilon_{n}^{\lambda+\varepsilon} s_{n}\left(\frac{t}{1+t}\right)^{n}
$$

is convergent for alz $t>0$, then

(1.4.2) $\sigma_{\lambda}(y)=\frac{\Gamma(\lambda+\varepsilon+1)}{\Gamma(\varepsilon) \Gamma(\lambda+1)} \frac{1}{y^{\lambda+\varepsilon}} \int_{0}^{y}(y-t)^{\varepsilon-1} t^{\lambda} \sigma_{\lambda+\varepsilon}(t) d t$ PROOF

By Lemma '1.1, $\sum_{n=0}^{\infty} \varepsilon_{n}^{\alpha} s_{n}\left(\frac{t}{1+t}\right)^{n}$ is absolutely convergent for all $\alpha>-1$, and for $t>0$. This justifies the interchange of the integration and summation signs in the righthand side of (1.4.2). The result then,follows immediately Erom Lemma 1.2 :

LEMMA 1.4

Suppose that

$$
\begin{aligned}
& \text { (i) } K(u, v) \geq 0 \text { for } u>0, v \geq 0 ; \\
& \text { (ii) } \int_{0}^{\infty} k(u, v) d v \rightarrow 1 \text { as } u \leq \infty \text { and } \int_{0}^{M} K(u, v) d v+0
\end{aligned}
$$

as $\mathrm{U} \rightarrow \infty$ for each $\mathrm{M}>0$;

(iii) $f(t)$ is continuous for $t>0$. 
Then, if $\mathrm{F}(\mathrm{u})=\int_{0}^{\infty} \mathrm{K}(\mathrm{u}, \mathrm{v}) \mathrm{f}(\mathrm{v}) \mathrm{dv}$ exists in the CauchyLebesgue sense for each $\mathrm{u}>0$, we have $\underset{v \rightarrow \infty}{\liminf } f(v) \leq \underset{u \rightarrow \infty}{\liminf } F(u) \leq \underset{u \rightarrow \infty}{\limsup } F(u) \leq \underset{v \rightarrow \infty}{\limsup } f(v)$.

Lemma 1.4 is the integral analogue' of Theorem 1.5 and is proved by an argument of the standard type. (cf. [ ] ], proof ọf Theorem 9).

Theorem 1.2 now follows immediately from Lemmas 1.3 and 1.4

Theorem $1.2^{C}$ and Lemmas 1.2 and 1.3 are due to Borwein [ 2 ]. Theorem 1.4 was proved by Borwein in 7$\}$ ] and was a corollary of a more general inclusion theorem on methods of summability básed on power sèries. We give a direct proof of Theorem 1.4 in Chapter 4 .

1.5 - ADDIFIOXYL SUMMABILITY METHODS. - C.ESARO SUNMABILITY. (c, $\alpha)$

We say that the sequence $\left\{s_{n}\right\}$ is summable to $s$ by the Cesaro method $(C, \alpha)$ for $\alpha>-1$, and write $s_{n} \rightarrow(C, \alpha)$ if

$$
s_{n}^{\alpha}=\frac{1}{\varepsilon_{n}^{\alpha}} \sum_{v=0}^{n} \varepsilon_{n-v}^{\alpha-1} s_{v} \rightarrow \text { s.as } n \rightarrow \infty \text {. }
$$

Set, for $y>0$

$$
r_{\alpha}(y)=\frac{1}{\Gamma(\alpha+1)} \frac{y^{\alpha}}{1+y} \sum_{n=0}^{\infty} s_{n}^{\alpha}\left(\frac{y}{1+y}\right)^{n}
$$

THE A $(\mathrm{C}, \alpha)$ METHOD

$$
\text { For } \alpha>-1 \text {, we say that's } s_{n} s A(C, \alpha) \text { if } \gamma_{\alpha}(y)
$$

converges for $y>0$ and $\lim _{y \rightarrow \infty} \Gamma(\alpha+1) y^{-\alpha} \Upsilon_{\alpha}(y)=s$. 
In connection with the $A(C, \alpha)$ method we state, without proof, the following known results.

.LEMMA 1.5

$$
\begin{gathered}
\text { If } y>0, \alpha>-1, \delta>0 \text { then } \\
\text { (1.5.1) } \gamma_{\alpha+\delta}(y)=\frac{1}{\Gamma(\delta)} \int_{0}^{y}(y-t)^{\delta-1} \gamma_{\alpha}(t) d t .
\end{gathered}
$$

For $\alpha \geq 0$, this result is due essentially to Kogbetliantz [9] (see also Lord [11], Borwein [4]). THEOREM 1.6

If $\alpha>-1, \delta>0$ then $A(C, \alpha) \subseteq A(C, \alpha+\delta)$.

This result, for $\alpha \geq 0$, is by Lord [11] and for $\alpha(>-1$ by Àmir [1] .

- THEOREM 1.7

$$
\text { If }-1<\alpha<1 \text {, then } A(C, \alpha) \simeq{ }^{A}-
$$

This result is due to Borwein [5]. As a corollary we observe that the $A(C, \alpha)$ method is regular, not only for $\alpha \geq 0$, but for $\alpha \geq-1$.

THE $(A, \lambda)$ METHOD.

Let $\left\{\lambda_{n}\right\}$ be a sequence of real numbers satisfying

$$
0 \leq \lambda_{1}<\lambda_{2}<\lambda_{3}<\cdots ; \quad \lambda_{n} \rightarrow \infty \text {. }
$$

The series $\sum_{n=1}^{\infty} a_{n}$ is said to be summable to $s$ by the" abelian summability method $(A, \lambda)$ if $\sum_{n=1}^{\infty} a_{n} e^{-y \lambda} n$ is.

convergent for $y>0$ and tends to $s$ as $y \rightarrow j:$. We then write $s_{n} \rightarrow s(A, \lambda)$.

With respect to the $(A, \lambda)$ method we observe that 


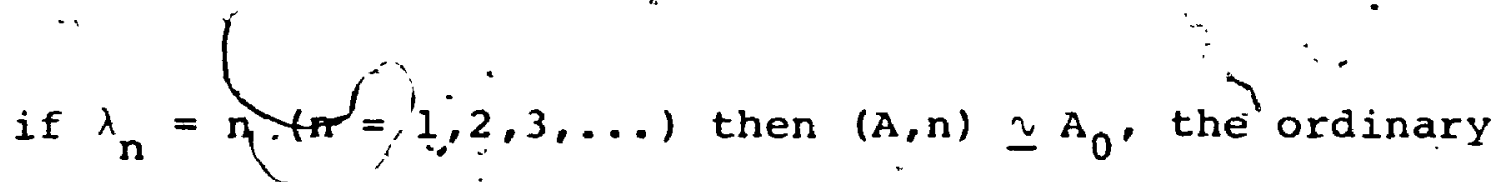
Abel method. In addition, it is well-known (see, for example, [7], Theorem 27) that the $(A, \lambda)$ method is regular.

1.6 REMARKS CONCERVING THEOPEMS 1.2 ari. 1.4

In this section we observe that the inclusions established in Theorems 1.2 and 1.4 , are strict inclusions, in the sense of the following theorems.

THEOREM 1.8

For $\lambda>\mu>-1$, there exists a segierce $\left\{\mathbf{s}_{\mathbf{n}}\right\}$ whici is $\left(A_{\mu}\right)$-convergent but not $\left(A_{\lambda}\right)$-eongergent.

PROOF

Let $\left\{s_{n}\right\}$ be the sequence such that $(1-x)^{\lambda+1} \sum_{n=0}^{\infty} E_{n}^{\lambda} s_{n} x^{n^{\prime}}=\sin \frac{1}{1-x}$ for $0 \leq x<1$. Now, the power series of $(1-x)^{-\lambda-1} \sin \frac{1}{1-x}$ is convergent for $0<x<1$, but since $\sigma_{\lambda}^{*}(y)=\sin (1+y)$ oscillates as $y+\infty,\left\{s_{n}\right\}$ is not $\left(A_{\lambda}\right)$-convergent.

However if $\lambda>\mu>-1$, we have by (1.4.2) that $\sigma_{\mu}(y)=\frac{f(\lambda+1)}{\Gamma(\mu+1) \Gamma(\lambda-\mu)} \frac{1}{y^{\lambda}} \int_{0}^{y}(y-t)^{\lambda-\mu-1} t^{\mu} \sin (1+t) d t$ $=\frac{\Gamma(\lambda+1)}{\Gamma_{i}(\mu+1) \Gamma(\lambda-\mu)} \int_{0}^{1}(1-u)^{\dot{\lambda}-\mu-1} u^{\mu} \sin (1+y u)$ +0 as. $y \rightarrow \infty$ by the Riemann-Lebesgue theorem. Hence $s_{n} \rightarrow O\left(A_{\mu}\right)$ and the progf is complete. 
This result is due to Borwein [ 2]. The following result is also due essentially to Borwein [4]. THEOREM $1.9 \%$

For $\lambda>-1$, there exists" a sequence $\left\{s_{n}\right\}$ which is L-convergent but not $\left(A_{\lambda}\right)$-convergent.

PROOF

Let $\left\{s_{n}\right\}$ be the sequence such that

$$
\sum_{n=0}^{\infty} s_{n} x^{n}=(1-x)^{-1-i}|x|<1 .
$$

Then $\gamma_{0}(y)=(1+y)^{i}$. Hence, by Lemma 1.5 , for $\lambda>0, y>0$ $\Gamma(\lambda) y^{-\lambda} \gamma_{\lambda}(y)=y^{-\lambda} \int_{0}^{y}(y-\dot{t})^{\lambda-1}(1+t)^{i} d t$

$=y^{-\lambda} \int_{-1}^{y}(y-t)^{\lambda-1}(1+t)^{i} d t-y^{-\lambda} \int_{-1}^{0}(y-t)^{\lambda-1}(1+t)^{i} d t$ $=\frac{\Gamma(\lambda) \Gamma(1+i)}{\Gamma(\lambda, i+1)} y^{-\lambda}(1+\dot{y})^{\lambda+i}-y^{-\lambda} \int_{-1}^{0}(y-t)^{\lambda-1}(1+t)^{i} d t$. Now, $y^{-\lambda} \int_{-1}^{0}(y-t)^{\lambda-1}(1+t)^{i} d t \rightarrow 0$ as $y \rightarrow \infty$. Hence $y^{-\lambda} \gamma_{\lambda}(y)$ does not tend to a limit as $y+\infty$, and thus $\left\{s_{n}\right\}$. is not $A(C, \lambda)$-convergent for $\lambda>0$. By Theorem 1.7, $\left\{s_{n}\right\}$ is not summable $\left(A_{\lambda}\right)$ for $-1<\lambda<0$ and hénce by Theorem 1.2. $\left\{s_{n}\right\}$ is not sumable $\left(A_{\lambda}\right)$ for any $\lambda>-1$ However, as $x \rightarrow 1$ in $(0,1)$, $\sum_{n=0}^{\infty} \frac{s_{n}}{n+1} x^{n+1}=\int_{0}^{x}(1-t)^{-1-i} d t=0[|\log .(1-x)|\}$ Hence $s_{n} \rightarrow O(L)$. This completes the proof. 
1

CHAPTER 2

a

A TÁÚBERIAN THEOREM

2.i THTRODUCTION

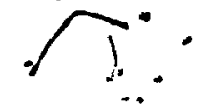

In this chapter we, introduce the main theorem, to follow in Chapter. Three, by giving an elementary proof. of the result in a special case.

\subsection{A DEFINITION}

The real-valued function, $f(x)$ is said to be slowly decreasing if for each $\varepsilon>0$, there exists a $\delta>0$ and an $M>0$, such that $f(y)-f(x)>-\varepsilon$ whenever $y \geq x \geq M$ and $0 \leq \ln \frac{y}{x}<\delta$.

Equivalently, $f(x)$ is slowly decreasing if

$$
\lim \inf \{f(y)-f(x)\} \geq 0
$$

whenever $\dot{y} \geq x \rightarrow \infty$ and $(y-x) / x \rightarrow 0$.

\subsection{STATEMENT OF THE THEOREM}

\section{THEOREM}

$$
\text { If } \lambda>-1, \sum_{n=0}^{\infty} a_{n}=s\left(A_{\lambda}\right) \text {, and } \sigma_{\lambda+1}(t) \text { is }
$$

slowly decreasing, then $\sum_{n=0}^{\infty} a_{n}=s\left(A_{\lambda+1}\right)$.

2.4 SOME LEMMAS

In this section we assume that $\lambda>-1$, that $f(t)$ is absolutely continuous on $[0, M]$ for each $M \geq 0$, and set: 
$(2.4 \cdot 1)$

$F(y)=\frac{\lambda+1}{y^{\lambda+1}} \int_{0}^{y} t^{\lambda} f(t) d t$

LEMMA 2.1

$$
\text { If } \mathrm{F}(\mathrm{y}) \rightarrow 0 \text { as } \mathrm{y} \rightarrow \infty \text {, then } \mathrm{f}(\mathrm{y}) \ddot{\rightarrow} \text { as } \mathrm{y} \dot{\rightarrow} \text { if and }
$$

$\cdots$, on ty if

$$
\frac{1}{y^{\lambda+1}} \int_{0}^{y} t^{\lambda+1} f^{\prime}(t) \cdot d t \rightarrow 0 \text { as } y+t_{\infty}^{t} \cdot
$$

13

PROOF,

An integration by. parts yields

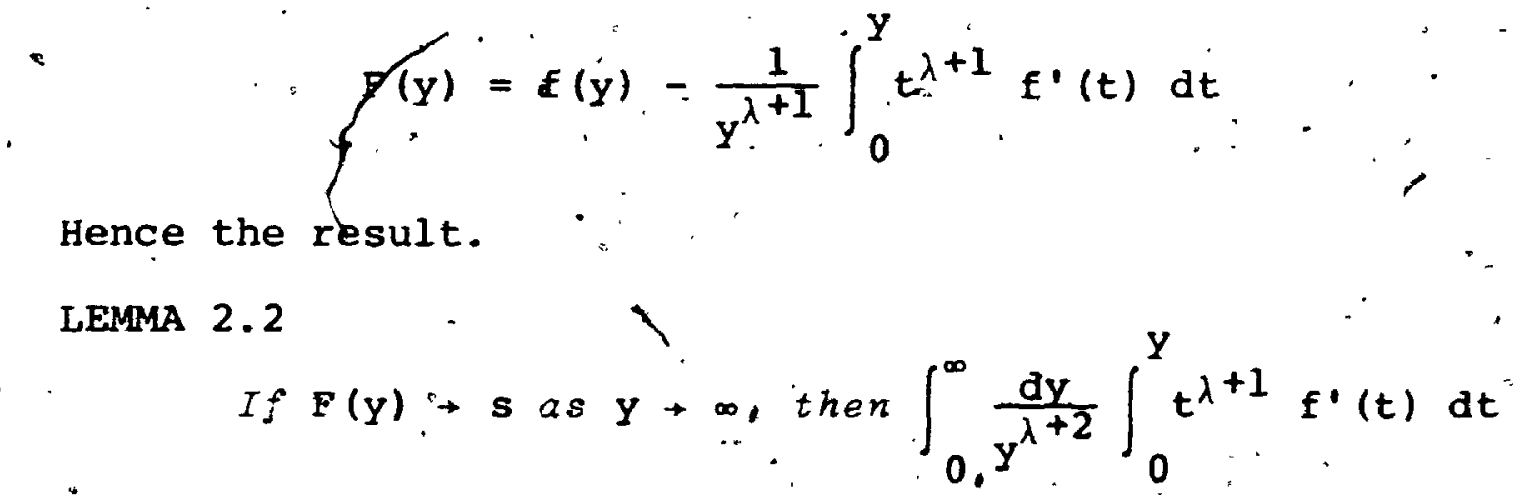

exists in the Cauchy-Lebesgue sense. PROOF

We have

$$
\begin{aligned}
& \int_{0}^{R} \frac{d y}{y^{\lambda+2}} \int_{0}^{y} t^{\lambda+1} f^{\cdot t}(t) d t=\int_{0}^{R} t^{\lambda+1} f^{\prime}(t) d t \int_{t}^{R} \frac{d y}{y^{\lambda+2}} \\
= & -\frac{1}{(\lambda+1) \cdot R^{\lambda} f} \int_{0}^{R} t^{\lambda+1} f^{\prime}(t) \ddot{d t}+\frac{1}{\lambda+1} \int_{0}^{R} f^{\prime}(t) d t \\
= & \frac{1}{R^{\lambda+1}} \int_{0}^{R} t^{\lambda} f(t) d t-\frac{f(R)}{\lambda+1}+\frac{f(R)}{\lambda+1}-\frac{f(0)}{\lambda+1} \\
\rightarrow & \frac{8-f(0)}{\lambda+1} \text { as } R \rightarrow \infty .
\end{aligned}
$$


The absolute continuity of $f(t)$ allows the inversion of the order of integration and the integration by parts performed here." The result follows.

LEMMA $2 \cdot 3$

If $\mathrm{f}(\mathrm{t})$. is slowly decreasing and $\mathrm{F}(\mathrm{y})^{\circ} \rightarrow 0$ as $\mathrm{y} \rightarrow \infty$ then $\mathrm{f}(\mathrm{Y})+0$ as $\mathrm{y} \rightarrow+\infty$.

PROOF

By Lemma 2.1 , it suffices to show

$$
\frac{1}{y^{\lambda+1}} \int_{0}^{y} t^{\lambda+1} E^{\prime}(t) d t \rightarrow 0 \text { as } y^{\prime} \rightarrow \infty \text {. }
$$

Suppose (2.4.2) does not hold. Then there exists a sequence $\left\{R_{n}\right\}$ tending to infinity and $a \cdot c>0$ such that either

$$
\int_{0}^{n} t^{\lambda+1} f^{\prime}(t) d t>c R_{n}^{\lambda+1} n=0,1,2, \ldots
$$

or

$$
\text { (ii) } \int_{0}^{R} t^{\lambda+1} f^{\prime}(t) d t<,-c R_{n}^{\lambda+1} \text { in }=0,1,2, \ldots
$$

Suppóse (i) holds.

since $f(t)$ is slowly decreasing, for a syffitiently small, but fixed, positive $\varepsilon, \operatorname{say} \frac{c}{4}>\varepsilon ">0$, there exists an $M>0$ and $\dot{a} \gamma>1$ such that $f(t),-f(x)>-\varepsilon$ whenever $x \geq M$ and $x \leq t \leq \gamma x$. Furthermore we may suppose $\gamma$ is. sma11 enough so that $\gamma^{\lambda+1} \varepsilon<\frac{C}{2}$.

Let $v$ satisfy the inequality $M \leq R_{n} \leq v^{\prime} \leq r R_{n} \cdot$ *

By the second mean value theorem for integrals, there exists a $u$ such that $\dot{R}_{n} \leq \digamma u v \leq r R_{n}$ and $\therefore \quad \int_{R_{i}}^{v} t^{\lambda+1} f^{\prime}(t) d t=v^{\lambda+1}\{f(v)-f(u)\}$. 
15

Hence

$$
\begin{aligned}
\int_{R_{n}}^{v} t^{\lambda+1} \cdot f^{\prime}(t) d t & >-\varepsilon \gamma^{\lambda+1} R_{n}^{\lambda+1} \\
& >-\frac{c}{2} R_{n}^{\lambda+1}
\end{aligned}
$$

"Therefore, when $\gamma R_{n} \geq Y \geq R_{n} \geq M$

$$
\begin{aligned}
& \int_{0}^{y} t^{\lambda+I^{\prime}} f^{\prime}(t) d t \\
= & \int_{0}^{R_{n}} t^{\lambda+1} f^{\prime}(t) d t+\int_{R_{n}}^{Y} t^{\lambda+1} f^{\prime}(t) d t \\
> & \frac{C}{2} R_{n}^{\lambda+1} .
\end{aligned}
$$

Hence, for $R_{n} \geq M$

$$
\begin{aligned}
\cdots & \int_{R_{n}}^{\gamma R_{n}} \frac{d y}{y^{\lambda+2}} \int_{0}^{y} t^{\lambda+1} f^{\prime}(t) d t \\
& \quad>\frac{C}{2} R_{n}^{\lambda+1} \cdot \int_{R_{n}}^{\gamma R_{n}} \frac{d y}{y^{\lambda+2}} \\
& =\frac{{ }_{x}}{2(\lambda+1)}\left\{1-\frac{1}{\gamma^{\lambda+1}}\right\} .
\end{aligned}
$$

Therefore, by Lemma $2.2, F \cdot(y)$ does not tend to a limit, which is a contradiction.

Suppose (ii) holds.

For $\frac{c}{2}>\varepsilon>0$, there exists an $M>0$ and $a$ sateseying $0<\delta<\neq$ such that $f(x)-f(t)>-\varepsilon$ whenever $x \geq M / \delta$ and $\delta x \leq t \leq x$. 
Now, for any $u$ satisfying $M \leq \delta R_{n} \leq u \leq R_{n}$, there exists, a $v$ satisfying $\delta R_{n} \leq u \leq v \leq R_{n}$ such that

$$
\begin{aligned}
\int_{u}^{R_{n}} t^{\lambda+1} f^{\prime}(t) d t & =R_{n}^{\lambda+1}\left\{f\left(R_{\Omega}\right)-f(v)\right\} \\
& >-\frac{C}{2} R_{n}^{\lambda+1} .
\end{aligned}
$$

Hence, for $M \leq \delta R_{n} \leq y \leq R_{n}$

$$
\begin{aligned}
& \int_{0}^{y} t^{\lambda+1} f:(t) d t \\
= & \int_{0}^{R} t^{\lambda+1} f \cdot(t) d t,-\int_{\dot{y}}^{R_{n}} t^{\lambda+1} f \cdot(t) d t \\
<-\frac{c}{2} \cdot R_{n}^{\lambda+1} &
\end{aligned}
$$

Therefore, when $M \leq \delta R_{n}$

$$
\begin{aligned}
& \int_{\delta R_{n}}^{R_{n}} \frac{d y}{y^{\lambda+2}} \int_{0}^{y} t^{\lambda+1} f^{\prime}(t) d t \\
& <-\frac{c}{2} R_{n}^{\lambda+1} \int_{\delta R_{n}}^{R_{n}} \frac{d y}{y^{\lambda+2}} . \\
& =-\frac{c}{2(\lambda+1)}\left\{\frac{1}{\delta^{\lambda+1}}-1\right\} .
\end{aligned}
$$

Again, by Lemma 2.2, $F(y)$ does not tend to a limit as $y \rightarrow \infty$, which is a contradiction. This establishes the result. 


\section{2:5 RROOF OF THEOREM 2.1}

It suffices to suppose that $s_{n} \rightarrow O\left(A_{\lambda}\right)$. By (1.4.2) we have

$$
\sigma_{\lambda}(y)=\frac{\lambda+1}{y^{\lambda+I}} \int_{0}^{y} t^{\lambda} \sigma_{\lambda+1} \text { (t) dt } y>0 .
$$

By hypothesis, $\sigma_{\lambda+1}(t)$ is slowly decreasing, and since $\sigma_{\lambda+1}(t)$ is absolutely continuous in $[0, x]$ for each $x \geq 0$, the result follows immediately from Lemma: 2.3.

2.6 COMMENT

Our object is to establish a more general version of Theorem 2.1 in which the occurrences of $(\lambda+1)$ in Theorem 2.1 are replaced by $(\lambda+\varepsilon)$ for an arbitrary, but fixed, $\varepsilon>0$. However, we adopt a different technique. of próf, since any generalization of the method of this chapter seems to be non-trivial. More specifically, the: integral transformation which would replace the $F(y)$ of this chapter adds extra difficulties. 

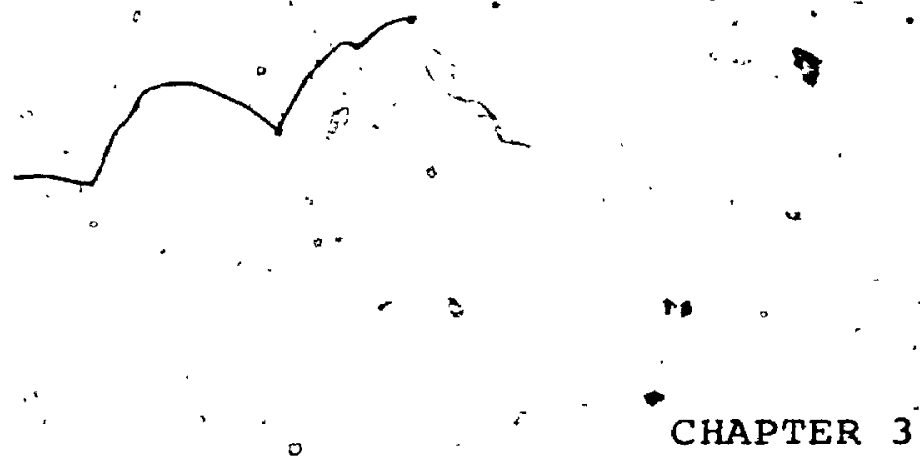

A TAUBERIAN THEOREM OF SLOWLY :DECREASING TYPE - I In this chapter we prove a taubierian theorem in the $\left(A_{\lambda}\right)$ scale, mope general, than Theorem 2.1. As a sṭep in that proof we also state and prove an jintegral analogue, with simplified.hypotheses, of a theorem initially proved by Vijayaraghavan [15] and also giver by Hardy in $[7]$. Finally, as,a corollary of the main result, we give $y$ tauberian theorêm of o-typé.

3.1 Ś:

The theorem we prove is the following.

THEOREM 3.1

$$
\text { For } \lambda>-\ln \text { and } \varepsilon>0, \text { if } \sum_{\mathbf{n}=0}^{\infty} a_{n}=s \cdot\left(A_{\lambda}\right) \text { and }
$$

$\sigma_{\lambda+\varepsilon}(t)$ is slowly decreä́ing, then $\sum_{n=0}^{\infty} a_{n}=s\left(A_{\lambda+\varepsilon}\right)$.

The proof is divided into two stages. In the first stage we show thot $\left(A_{\lambda}\right)$ summability, and the fact that $\sigma_{\lambda+E}(t)$ is slowly decreasing imply that $\sigma_{\lambda+\varepsilon^{\prime}}(t)$ is bounded.

This.wil! follow by the vijayaraghavan-type theorem in the next sectigp. In the second stage we go from the boundedness of : $\sigma_{\lambda+\varepsilon}(t)$ to $\left(A_{\lambda+\varepsilon}\right)$ summability, by means of a wiener tauberian theorem: 
3.2 A VIJAYARAGHAVAN-TYPE RESULT

$f$ In the next three sections we assume the following initial hypotheses.

(i) $K(u, v)$ is defined, real-valued, and non-negative*. for $u>0, v \geq 0.0^{\circ}$ Moreover $\int_{0}^{\infty} k(u, v) d v$ exists in the sense of Lebesgue for each $\bar{u}>0$ :

(ii) $\int_{0}^{\infty} k(u, v) d v ; 1$ as $u \neq \infty$

(iii) $f(v)$ is real-valued and continuous for $v \geq 0$. (iv) $F(u)=\int_{0}^{\infty} K(u, v) f(v) d v$ exists in the Cauchy-

Lebesguè sense for each a $>0$. THEÖREM 3.2

Suppose the following conditions hold:

(3.2.1) $\Phi(\mathbf{x})$ is a real-valued, non-negative, increasing, - continuous function defined on $[0, \infty)$ such that $\Phi(x)$ qs $\mathrm{x} \rightarrow{ }^{\infty}$;

- (3.2.2) liminf $\{f(y)-f(x)\} \geq-\mu>-\infty$ for some fixed; positive $u$, whenever $y>x \rightarrow \infty_{0}$ and $\phi(y)-\Phi(x) \rightarrow 0_{B}$

$.13 .2 .3) \Phi(x)-\Phi(x-1) \rightarrow 0$ as $x \rightarrow \infty$

(3.2.4) $\int_{0}^{\dot{x}} x(u, v) d v \rightarrow 0$ whenever $u>x \rightarrow \infty$ and

$=\Phi(u)-\Phi(x) \rightarrow \infty$;

$(3.2 .5) \int_{x}^{\infty} \mathrm{K}(\mathrm{u}, \mathrm{v})\{\Phi(\mathrm{v})-\Phi(\mathrm{x})\} \mathrm{dv}+0$ whenever $\mathrm{x}: \mathrm{u} \rightarrow \infty$ and $\dot{\phi}(\mathrm{x})-\phi(\mathrm{u})+\infty$; and 
(3.2.6) $F(u)=O(1)$ for $u \ngtr 0$ Then $f(v)=O(1)$ for $v>0$.

3. 3 SOME PRELIMINARY LEMMAS LEMMA 3.1

If $(3.2 .1)$ and $(3.2 .4)$ hold, then $f(\hat{v}) \rightarrow$ s as $v, \rightarrow \infty$ implies $\mathbf{F}(\mathrm{u}) \rightarrow \mathbf{s}$ as $\mathbf{u} \rightarrow \infty$ for finite or infinite $\mathbf{s}$. PROOF

By Lemma $I^{\circ} .4$ it suffices to show that for every fixed $M>0$.

$$
\int_{0}^{M} K(u, v) d v+p \text { as } u+\infty
$$

Take $\varepsilon>0$. By $(3.2,4)$ there exist an $x_{1} \geq \mu>0$ and an Rfi 0 such that.

$$
\int_{0}^{x_{1}}-K(u, v) d v \leq \varepsilon \text { whenever }
$$

$u>x_{1}$ and $\Phi(u)-\Phi\left(x_{1}\right) \geq R_{1}$. Define $u_{1}$ to be that value of $u$-such that $\phi\left(U_{1}\right)=\dot{R}_{1}+\Phi\left(x_{1}\right)$. Then for $u \geq v_{1}$.

$$
\int_{0}^{M} K(u, v) d v \leq \int_{0}^{x_{1}} k(u, v) d v<\varepsilon
$$

This completes the proof.

- LEMMA 3.2

$$
\text { If (3.2.1) and (3.2.2) hold, then there exist }
$$

positive constants $M_{1}, M_{2}$ uch that

$f(y)-f(x)>-M_{1}\{(y)-\phi(x)\}-M_{2}$ for $\left.y \geq x \geq 0.\right\rangle$

PROOF

Take $\varepsilon_{0}>0 . \quad$ By $\left.\times 3.2 .2\right)$ there exist $x_{1}>0$ and 
$\dot{\delta}>g$ such that

(3.3.1) $f(y)-f(x)>-\mu-\varepsilon$ whenever $y>x \geq x_{1}$ and $\Phi(y)-\Phi(x) \leq \delta$.

But there exists an $M_{2}>0$ such that $f(y)-f(x)>-M_{2}$ whenever $y>x \geq 0$ and $\Phi(y)-\Phi(x) \leq \delta$. For if

(i) $x_{1} \geq y>x \geq 0$ then $y$ is clearly bounded above.

If

(ii) $y>x_{1} \geq x \geq 0$ and $\phi(y)-\Phi(x) \leq \delta$ then

$\Phi(y)-\Phi\left(x_{1}\right) \leq \Phi(y)-\Phi(x) \leq \delta$ and since $\Phi$ is increasing to infinity, $y$ is bounded above. In either case; since $f(x)$ is continuous, there exists a positive iv such that $f(y)-f(x)>-N_{1} \cdot$ Combining this with. (3.3.1) we take $M_{2}=\operatorname{MAx}\left\{\mu+\varepsilon, N_{1}\right\}$ to get $f(y)-f(x)>-M_{2}$ whenever $y>x \geq 0$ and $\Phi(y)-\Phi(x) \leq \delta$. Now fix $x, y$ such that $y>x . \geq 0$. Define a sequence $\left\{x_{r}\right\}$ for $r=0,1,2 ; \ldots$ such that $x_{0}=x$ and $x_{r}$ satisfies $\Phi\left(x_{r}\right)=\Phi\left(x_{r-1}\right)+\delta \quad r=1,2, \ldots$. since $\Phi\left(x_{r}\right)=\Phi\left(x_{0}\right)+r \delta$ we have $x_{r} \rightarrow \infty$ às $r \rightarrow \infty$. Hence, there exists an integer m such that $x_{m} \leq y<x_{m+1}$. Therefore

$$
\text { (3.3.2) } \begin{aligned}
f(y)-f(x) & =\sum_{x=0}^{m-1}\left\{f\left(x_{r+1}\right)-f\left(x_{r}\right)\right\}+f(y)-f\left(x_{m}\right) . \\
& >-m M_{2}-M_{2} .
\end{aligned}
$$

Hówever, since $m \delta=\Phi\left(x_{m}\right)-\Phi\left(x_{0}\right) \leq \Phi(y)-\Phi(x)$ we have

$$
-m \geq-\frac{\Phi(x)-\Phi(x)}{\delta}
$$

Therefore by $(3.3 .2)$

$$
E(y)-f(x)>-\frac{M_{2}}{\delta}\{\Phi(y)-\Phi(x)\}-M_{2}
$$

The desired resylt follows. 
22

LEMMA

$\cdot$

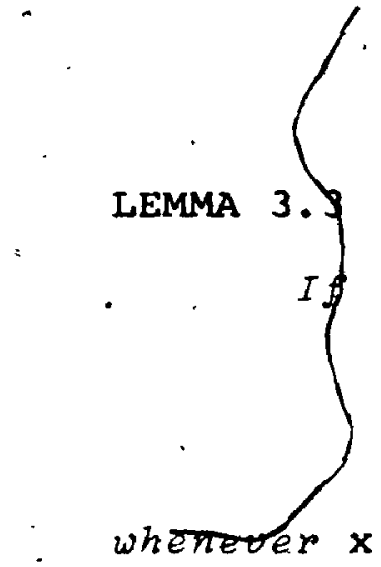

I\$ (3.2.1) and (3.2.5) hold, then

$$
\int_{x}^{\infty} k(u, v) d v \rightarrow 0
$$

Take $\varepsilon>0$. By $(3.2 .5)$, there exist an $x_{0}>0$ and $R_{0}>$ such that

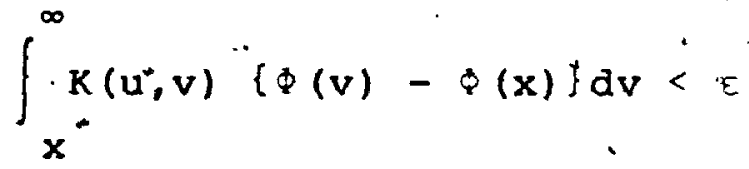

whenever $x>u>x_{0}$ and $\Phi(x)-\Phi(u) \geq R_{0}$. Now take fixed $x$ and $u$ such that

$$
x>u>x_{0} \text { and } \Phi(x)-\Phi(u) \geq R_{0}+1
$$

Since $\Phi(x)$ is continuous and increasing, there exists a $w$ $\therefore$ satisfying $u<w<x$ and $\Phi(x)-\Phi(w)=1$. Now

$$
{ }^{\prime} \Phi(w)-\Phi(u)=\Phi(w)-\Phi(x)+\Phi(x)-\Phi(u) \geq-1+R_{0}+1=R_{0}
$$

$\because \quad$ Hence

$f$

$\cdot$

$$
\therefore \quad \int^{\infty} k(u, v) d v=\int^{\infty} k(u, v)[\Phi(x)-\Phi(w)\} d v
$$

$$
\leq \int_{x}^{\infty} k(u, v)\{\Phi(v)-\Phi(w)\} d v
$$

$$
\leq \int^{\infty} K(u, v)\{\Phi(v)-\Phi(w)\} d v<\varepsilon
$$

This completes the proof? $\therefore$ 


\subsection{PROOF OF THEOREH 3.2}

PROOF

Set

$$
\begin{aligned}
& \alpha_{1}(t)=\sup _{0 \leq v \leq t} f(v) \\
& \alpha_{2}(t)=\sup _{0 \leq v \leq t}(-f(v))
\end{aligned}
$$

Suppose $f(t)$ is not bounded. Then

$$
\text { (3.4.1) } \underset{t \rightarrow \infty}{\limsup }|f(t)|=\infty
$$

If $f(t) \rightarrow \infty$ as $t \rightarrow \infty$, then, by Lemma $3.1, F(u) \rightarrow \infty$ as $u \rightarrow \infty$, which is a contradiction of (3.2.6). Similarly, if $f(t) \rightarrow-\infty$ as $t \rightarrow \infty$, then $F(u) \rightarrow-\infty$ as $u \rightarrow \infty$. By $(3.4 .1)$ at least one of $a_{1}$ or $a_{2}$ tends to infinity. Moreover, either (ij) or (ii) below holds.

(i) There exists a sequence $\left\{t_{k}\right\}(k=1,2, \ldots)$, tending to infinity, such that $\alpha_{1}\left(t_{k}\right) \geq \alpha_{2}\left(t_{k}\right) k=1,2, \ldots$

(ii) There exists a $t_{0}>0$ such that $\alpha_{2}(t)>\alpha_{1}(t)$ for all $t>t_{0}$. Case (i). We must have $a_{1}(t) \rightarrow \infty$ as $t \rightarrow \infty$. Furthermore, the sequence $\left\{t_{k}\right\}$ may be chosen such that (3.4.2) $f\left(t_{k}\right)=\alpha_{1}\left(t_{k}\right) \geq \alpha_{2}\left(t_{k}\right) k=1,2 \ldots$. For, if $v_{k}$ satisfies $0 \leq v_{k} \leq t_{k}$ and $f\left(v_{k}\right)=\alpha_{l}\left(t_{k}\right)$, then, since $\alpha_{1}$ and $\alpha_{2}$ are increasing, and $f$ is continuous we have

$$
\alpha_{1}\left(v_{k}\right)=f\left(v_{k}\right)=\alpha_{1}\left(t_{k}\right) \geq \alpha_{2}\left(t_{k}\right) \geq \alpha_{2}\left(v_{k}\right)
$$

Hence, we may replace $t_{k}$ by $v_{k}$, and the new sequence clearly tends to infinity. 


$$
\text { For any } R>0 \text {, let, } x=x(R) \text { represent. }
$$

$\inf \left\{t_{k} \mid \alpha_{1}\left(t_{k}\right) \geq \alpha_{2}\left(t_{k}\right)\right.$ and $\left.\alpha_{1}\left(t_{k}\right)>2 R\right\}$.

set

$\Delta(R)=\left\{t \mid t^{\prime}>x(R)\right.$ and $\left.f(t) \leq f(x) / 2\right\}$.

Now, since $f(t)$ does not tend to infinity, $\Delta(R) \neq$ for large $R$. "Let $y=y(R)=$ inf $\Delta(R)$. For any $t \epsilon \Delta(R)$, by Lermia 3.2 .

\section{Hence}

$$
\longrightarrow f(t)-f(x)>-M_{1}\{\phi(t)-\Phi(x)\}-M_{2} \text {. }
$$

$$
\begin{aligned}
M_{1}\{\Phi(t)-\Phi(x)\} & >f(x)-f(t)-M_{2} \\
& \geq \frac{f(x)}{2}-M_{2} \\
& >R-M_{2} \cdot
\end{aligned}
$$

Therefore, by the continuity of $\Phi, \Phi(y)-\Phi(x) \rightarrow \infty$ as $R \rightarrow \infty$ and for sufficiently large $R, y>x$.

Let $u \in(x, y)$ satisfy

$$
\Phi(u)=\{\Phi(x)+\Phi(y)\} / 2 \text {. }
$$

It is. immediate that $\phi(u)-\phi(x) \rightarrow \infty$ and $\delta(y)-\phi(u) \rightarrow \infty$ as $R \rightarrow \infty$. Write

$$
\begin{aligned}
F(u) & =\left(\int_{0}^{x}+\int_{x}^{y}+\int_{y}^{\infty}{ }^{x} k(u, v) f(v) d v\right. \\
& =I_{1}(u)+I_{2}(u)+I_{3}(u)
\end{aligned}
$$

and consider each of $I_{1}, I_{2}, I_{3}$ in turn.

$$
\text { For } 0 \leq v \leq x,-f(v) \leq \sup _{0 \leq t \leq x}(-f(t))^{\circ}=a_{2}(x) .
$$

Hence $f(v) \geq-a_{2}(x)$, for $0 \leq v \leq x$ and 
(3.4.3) $I_{1}(u) \geq-\alpha_{2}(x) \int_{0}^{x} x(u, v) d v$

$$
\begin{aligned}
& \geq-\alpha_{1}(x) \int_{0}^{x} k(u, v) d v \\
& =-\alpha_{1}(x) \circ(1) \text { as } R \rightarrow \infty
\end{aligned}
$$

by $(3.2 .4)$.

Consider $I_{2}(u)$. We have

$$
\begin{aligned}
(3.4 .4) I_{2}(u) & =\int_{x}^{y} K(u, v) f(v) d v \\
& \geq \frac{f(x)}{2} \int_{x}^{y} K(u, v) d v \\
& =\frac{\alpha_{1}(x)}{2}\left\{1-o(1)-\int_{0}^{x} K(u, v) d v-\int_{y}^{\infty} K(u, v) d v\right\} \\
& =\alpha_{1}(x)\{1 / 2-O(i)\} \text { as } R \rightarrow \infty
\end{aligned}
$$

by (3.2.4) and Lemma 3.3 .

For $I_{3}(u)$, suppose $v>y>1$. Then $f(v)-. f(y-1)>-M_{1}\{\Phi(v)-\Phi(y-1)\}-M_{2}$. But $f(y-1)>\frac{f(x)}{2}>R>M_{2}+1$ for large enough $R$. Hence, $f(v)>-M_{1} \Phi(v)+M_{1} \Phi(y-1)+1$. By (3.2.3), for $R$ large enough $\Phi(y)-\Phi(y-1)<1 / M_{1}$. That is $M_{1} \Phi(y-l)+1>M_{1} \Phi(y)$. Hence $f(v)>-M_{1}\{\Phi(v)-\Phi(y)\}$. Therefore. 


$$
\begin{aligned}
(3.4: 5) I_{3}(u) & =\int_{Y}^{\infty} K(u, v) f(v) d v \\
& >-M_{1} \int_{y}^{\infty} K(u, v)\{\Phi(v)-\Phi(y)\} d v \\
& =-0(1) \text { as } R \rightarrow \infty .
\end{aligned}
$$

Combining $(3.4 .3),(3.4 .4)$, and (3.4.5) we have

$$
\begin{aligned}
F(u) . & >\alpha_{1}(x)\{1 / 2-0(1)\}=0(1) \\
& \rightarrow \infty \text { as } R \rightarrow \infty
\end{aligned}
$$

since $\alpha_{1}(t) \rightarrow \infty$ as $t \rightarrow \infty$.

This is in contradiction of $(3.2 .6)$.

Case (ii). We have $\alpha_{2}(t)>\alpha_{1}(t)$ for $t>t_{0}$. This implies that $\alpha_{2}(t) \rightarrow \infty$ as $t . \rightarrow \infty$ Take $R>-0$ and let $y=y(R)=\inf \left\{t \mid t>t_{0}\right.$ and $\left.{ }^{*} a_{2}(t)=f(t) \leq-2 R\right\}$.

set

$\Delta(R)=\left\{t \mid 0 \leq t \leq y\right.$ and $\left.f(t)>f(y) / 2=-\alpha_{2}(y) / \dot{2}\right\}$.

Note that $\Delta(R)$ is non-empty for large $R$ since $f(y)^{*} \rightarrow-\infty$ as $R \rightarrow \infty$. Let $x=x(R)=\sup \Delta(R):$ Now $x(R) \rightarrow \infty$ as $R \rightarrow \dot{\infty} \cdot$ Let $t \in \Delta(R)$. Then, by Lemma 3.2

$$
\begin{aligned}
M_{1}\{\Phi(y)-\Phi(t)\} & >f(t)-f_{0}(y)-M_{2} \\
& >-\frac{f(y)}{2}-M_{2 *}
\end{aligned}
$$

Now, $-f(y) / 2 \geq$. R. Hence,

$$
M_{1}\{\Phi(y)-\Phi(x)\} \geq R-M_{2}
$$

and it follows that $x<y$ and $\Phi(y)-\Phi(x) \rightarrow \infty$ as $R \rightarrow \infty$ 
Let $u . \epsilon(x, y)$ satisfy

$$
\Phi(\mathrm{u})=\{\Phi(\mathrm{x})+\Phi(\mathrm{y})\} / 2
$$

Then $\Phi(y)-\Phi(u)+\infty$ and $\Phi(u)-\Phi(x) \rightarrow \infty$ as $R \rightarrow \infty$.

We again write

$$
\begin{aligned}
F(u) & =\left(\int_{0}^{x}+\int_{x}^{y}+\int_{y}^{\infty} \int_{(u, v)} f(v) d v\right. \\
& =-I_{1}(u)+I_{2}(u)+. I_{3}(u)
\end{aligned}
$$

Then,

$$
\begin{aligned}
I_{1}(u) & =\int_{0}^{x} k(u, \ddot{v}) f(v) d v \\
& \leq a_{1}(x) \int_{0}^{x} k(u, v) d v \\
& \leq a_{2}(x) \int_{0}^{x} k(u, v) d v \\
& \leq a_{2}(y) \text {. } \quad \text { as } R \rightarrow \infty .
\end{aligned}
$$

\section{Hence}

$$
\text { When } x<v<y, f(v) \leq-a_{2}(y) / 2
$$

$$
\begin{aligned}
I_{2}(u) \leq-\frac{a_{2}(y)}{2} \int_{x}^{y} k(u, v) d v \\
\quad=-\frac{a_{2}(y)}{2}\left\{1-o(1)-\int_{0}^{x} k(u, v) d \dot{v}\right. \\
\ldots
\end{aligned}
$$$$
::=-\alpha_{2}(y)\{1 / 2-\infty(1)\} \text { as } R \rightarrow \infty
$$ 
It remains to consider $I_{3}(u)$. When $v>t_{0}$ we have. $f(v) \leq \alpha_{1}(v) \leq \alpha_{2}(v)$. Hence.

$$
\begin{aligned}
I_{3}(u) & \leq \int_{y}^{\infty} K(u, v) \alpha_{2}(v) d v \\
& =\int_{y}^{\infty} K(u, v)\left\{\alpha_{2}(v)-\alpha_{2}(y)+\alpha_{2}(y)\right\} d v .
\end{aligned}
$$

We now show that for $v>y$

(3.4.6) $\alpha_{2}(v)-\alpha_{2}(y)<M_{1}\{\phi(v)-\Phi(y)\}+M_{2}:$

First, for $v>y$

$$
\begin{aligned}
-f(v)-\alpha_{2}(y) & =-f(v)+f(y) \\
& =-(f(v)-f(y)) \\
& <M_{1}\{\Phi(v)-\Phi(y)\}+M_{2}
\end{aligned}
$$

Now $\alpha_{2}(v)=\max \left\{\alpha_{2}(y), \sup _{y \leq t \leq v}(-f(t))\right\}$. If $\alpha_{2}(v)=\alpha_{2}(y)$, the left side of $(-3.4 .6)$ "Is zero, and we are done. Otherwise, ${ }^{\prime} \alpha(v)=-f\left(t_{1}\right)$ where $y<t_{1} \leq v$. Thein.

$$
\begin{aligned}
\alpha_{2}(v)-\alpha_{2}(y) & <M_{1}\left\{\Phi\left(t_{1}\right)-\Phi(y)\right\}+M_{2} \\
& \left.\leq M_{1}\{\Phi(v)-\Phi(y)\}+M_{2}\right\}
\end{aligned}
$$

and $(3.4 .6)$ follows.

Hence

$$
\begin{aligned}
& \quad I_{3}(u) \leq M_{1} \int_{y}^{\infty} K(\dot{u}, v)\{\Phi(v)-\Phi(y)\} d v \\
& \quad+M_{2} \int_{y}^{\infty} k(u, v) d v+\alpha_{2}(y) \int_{y}^{\infty} K(u, v) d v \\
& \quad=a_{2}(y) \circ(1)+o(1) a s R \rightarrow \infty
\end{aligned}
$$


$\therefore \quad$ It follows that

$$
\begin{aligned}
F(u) & \leq-\alpha_{2}(y)\{1 / 2-o(1)\}+o(1) \\
& \rightarrow-\infty \text { as } R \rightarrow \infty
\end{aligned}
$$

This is a contradiction of $(3.2 .6)$ and the theorem " follows.

$\because 3.5$ A TAUBERIAN THEOREM OF WIENER

In this section we state a version of a theorem due to Wiener and found in [7], [18] and [16].

THEORÉM 3.3

$$
\text { If }
$$

(3.5.1) $g(t)$ is, Lebesgue integrable over the open interval $(0, \infty)$,

(3.5.2) $\int_{0}^{\infty} g(t) t^{-i x} d t \neq 0$ for any real $x$,

(3.5.3) $f(t)$ is slowly decreasing,

. (3.5.4) $f(t)$ is bounded for $t \geq 0$, and

$(3.5 .5) \cdot \lim _{y \rightarrow \infty} 1 / y \int_{0}^{\infty} g(t / y) f(t) d t$

$$
=s \int_{0}^{\infty} g(t) d t
$$

then $\mathrm{f}(\mathrm{t}) \rightarrow \mathrm{s}$ as $\mathrm{t} \rightarrow \infty$.

,

3.6 PROOF OF THEOREM 3.1

By (1.4.2) we have

$\sigma_{\lambda}(y)=\frac{\Gamma(\lambda+\varepsilon+1)}{\Gamma(\lambda+1) \Gamma(\varepsilon)} \frac{1}{y} \int_{0}^{y}(1-t / y)^{\varepsilon-1}(t / y)^{\lambda} \sigma_{\lambda+\varepsilon}(t) a t$.

It suffices to show that the conditions of 
Theorrem 3.3 are satisfied with $g(t)=\frac{\Gamma(\lambda+\varepsilon+1)}{\Gamma(\lambda+1) \Gamma(\varepsilon)} t^{\lambda}(1-t)^{\varepsilon-1}$, for $0<t<1$, and zero otherwise, and $f(t)=\sigma_{\lambda+\varepsilon}(t)$.

Note that $\int_{0}^{\infty} K_{g}(t) d t=1$.

Clearly, (3.5.1) and (3.5.2) hold. Also, (3.5.3)

and $(3.5 .5)$ hold by hypotheses of Theorem 3.1 . It remains to show that $f(t)$ is bounded for. $t \geq 0$.

It suffices to show that the conditions and initial hypotheses of Theorem 3.2 are satisfied.with

$K(u, v)=\frac{\Gamma(\lambda+\varepsilon+1)}{\Gamma(\lambda+I) \Gamma(\varepsilon)} \frac{1}{u}\left(\frac{v}{u}\right)^{\lambda}\left(1-\frac{v}{u}\right)^{\varepsilon-1}$, for $0<v<u$, and zero otherwise, and $\Phi(x)=x / e$, for $0 \leq x \leq e$, and

$\Phi(x)=\ln x$ for $e<x<\infty$.

Now, $k(u, v) \geq 0$ and $\int_{0}^{\infty} k(u, v) d v=1$ for $u>0$.

Moreover, since $f(v)$ is continuous, $\int_{0}^{\infty} k(u, v) f(v) d v$ exists for each $u>0$.

Conditions $(3.2 .1),(3.2 .2) \times(3.2 .3)$ and $(3.2 .6)$

are evidently satisfied. Also, since $K(u, v)=0$ whenever $v \geq u>0,(3.2 .5)$ holds. It remains to show that $(3.2 .4)$ holds. For $u>x$ we have

$$
\begin{aligned}
\int_{0}^{x} K(u, v) d v & =\frac{M}{u} \int_{0}^{x}\left(\frac{v}{u}\right)^{\lambda}\left(1-\frac{v}{u}\right)^{\varepsilon-1} d v \\
& =M \int_{0}^{x / u} t^{\lambda}(1-t)^{\varepsilon-1} d t \\
& \rightarrow 0 a s x / u \rightarrow 0
\end{aligned}
$$

and hence as $\ln \frac{\mathrm{u}}{\mathrm{f}} * \infty$. 
This completes the proof of Theorem 3.1 .

\subsection{A TAUBERIAN THEOREM OF O-TYPE}

In this section we prove the following result:

THEOREM $3 . .4$

$$
\begin{aligned}
& \text { For } t>0 \text { aind } \mu>\lambda>-i, \text { if } \sum_{n=0}^{\infty} a_{n}=s_{i}\left(A_{\lambda}\right) \text { and } \\
& \sigma_{\mu}(t)=0(1) \text {, then } \sum_{n=0}^{\infty} a_{n}=s\left(A{ }_{\lambda+\delta}{ }^{\prime} \text { for } 0 \leq \delta<\mu-\lambda .\right.
\end{aligned}
$$

The proof will be deduced from Theorem 3.1 and two additional lemmas.

- LEMMA 3.4.

If $\mathrm{f}$ is Lebesgue-integrable on the interval $(-\infty, \infty)$, then

$$
\text { 1 } \int_{-\infty}^{\infty}|f(r x)-f(x)| d x+0 \text { as } r+1
$$

- PROOF

Take $\varepsilon>0$, and suppose $\frac{1}{2} \leq r^{*} \leq 2$. For any $M>0$,

$$
\begin{aligned}
& \int_{-\infty}^{-M}|f(r x)-f(x)| d x+\int_{M}^{\infty}\left|f_{0}(r x)-f(x)\right| d x \\
& \leq \frac{1}{r} \int_{-\infty}^{-r M}|f(x)| d x+\int_{-\infty}^{-M}|f(x)| d x \\
& +\frac{1}{r} \int_{r M}^{\infty}|f(x)| d x+\int_{M}^{\infty}|f(x)| d x \\
& \leq 3 \int_{-M / 2}^{\infty}|f(x)| d x+3 \int_{M / 2}^{\infty}|f(x)| d x
\end{aligned}
$$

Now, since $f(x) \in L(-\infty, g)$, there exists an $M_{0}>0$, such that whenever' $M>M_{0}$ 


$$
3 \int_{-\infty}^{-M / 2}|f(x)| d x+3 \int_{M / 2}^{\infty}|f(x)| d x<\varepsilon / 2 .
$$

Furthermore, since $f(x) \in \mathrm{L}(-\infty, \infty)$, there exists a function $g(x)$, continuous on the real line, such that

$$
\int_{-\infty}^{\infty}|f(x)-g(x)| d x<\frac{\hat{\varepsilon}}{0} / 12 \text {. }
$$

For a fixed $M>M_{0}$, since $g(x)$ is uniformly continuous on the interval $[-2 M, 2 M]$, there exists a $\delta_{1}>0$ such that<smiles>CCC</smiles>

$$
\because \quad\left|g(r x)-g\left(x^{*}\right)\right|<\frac{\varepsilon}{12 M} .
$$

whenever $|r x-x|<\delta_{1}$ and $x \in[-M, M]$ (hence $r x \in[-2 M, 2 M]$ ). Let $\delta \stackrel{m i n}{=}\left\{\frac{1}{M}, \frac{1}{2}\right\}$. Then, whenever $x \in[-M, M]$ and $|r-1|<\delta$,

$$
\int_{-M}^{M}|f(r x)-f(x)| d x
$$$$
\leq \int_{-M}^{M}|f(r x)-g(r x)| d x+\int_{-M}^{M}|g(r x)-g(x)| d x
$$$$
f \int_{-M}^{M}|g(x)-f(x)| d x \text {. }
$$

$$
\begin{aligned}
& \leq \frac{1}{r} \int_{-r M}^{r M} \mid f(x)-g(x) \int d x+\frac{\varepsilon}{12 M}(2 M)+\frac{\varepsilon}{12} \\
& <\frac{\varepsilon}{6}+\frac{\varepsilon}{6}+\frac{\varepsilon}{6}=\frac{\varepsilon}{2} . \quad \therefore .
\end{aligned}
$$

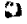


33

Therefore, $\int_{-\infty}^{\infty}|f(r x)-f(x)| d x<\varepsilon$ whenever $|r-1|<\delta$.

9

This completes the proof.

LEMMA 3.5

- If

(3.7.1) $h(t) \in L(0,1)$, and

(3.7.2) $f(t)$ is measurable and botinded fort $\geq 0$, then

a the function $\mathrm{F}(\mathrm{y})$, defined for $\mathrm{y}>0, b_{y}$

$$
F(y)=\frac{1}{y} \int_{0}^{y_{0}} h(t / y) f(t) d t \text { is slowly decreasing. }
$$

It suffices to show for $y>x>0$, that

$$
\begin{aligned}
& |F(y)-F(x)| \rightarrow 0 \text { as } y / x+1 \\
& |F(y)-F(x)| \\
= & \left|\frac{1}{y} \int_{0}^{y} h(t / y) f(t) d t-\frac{1}{x} \int_{0}^{x} h(t / x) f(t) d t\right| \\
= & \mid \frac{1}{y} \int_{0}^{x} h(t / y) f(t) d t-\frac{1}{x} \int_{0}^{x}\{h(t / y)+h(t / x)-h(t / y)\} f(t) d t
\end{aligned}
$$

$$
+\frac{1}{y} \int_{x}^{y} h(t / y) f^{\prime}(t) d t
$$$$
\leq \frac{M(y-x)}{x y} \int_{0}^{X}|h(t / y)| d t+\frac{M}{X} \int_{0}^{x}|h(t / y)-h(t / x)|_{0} d t
$$

$$
+\frac{M}{y} \int_{x}^{y}|h(t / y)| d t
$$


$=M\left(\frac{y}{x}-1\right) \int_{0}^{x / y}|h(t)| d t+M \int_{0}^{l}\left|h\left(\frac{x}{y} t\right)-h(t)\right| d t$

$$
+M \int_{x / y}^{1}|h(t)| d t
$$

+0 as $x / y \rightarrow 1$ by Lemma 3.4 and $(3.7 .1)$.

This completes the proof of the lemma.

PROOF OF THEOREM 3.4 .

By Theorem 3.1, it suffices to show that $\sigma_{\lambda+\delta}(t)$ is slowly decreasing. By $(1.4 .2)$ we have

$$
\sigma_{\lambda+\delta}(y)=\frac{1}{y} \int_{0}^{y} h(t / y) \sigma_{\mu}(t) d t
$$

where $h(t)=\frac{\Gamma(\mu+1)}{\Gamma(\mu-\lambda-\delta) \Gamma(\lambda+\delta+1)} t^{\lambda+\delta}(1, t)^{\mu-\lambda-\delta-1}$

for $0<t<1$ and is zero otherwise. Then since $\sigma_{\mu}^{\prime}(t)$ is bounded for $t \geq 0$ and $h(t) \in L(0,1)$, the result foliows by Lèma 3.5 .

NOTE. If $\left\{s_{n}\right\}$ is the sequence defined

by $(1-x)^{\mu+1} \sum_{n=0}^{\infty} \varepsilon_{n}^{\mu} s_{n}^{2} x^{n}=\sin \frac{1}{1-x}$ for $|x|<1$, then

$\sigma_{\mu}(t)=\sin (1+t)$ is bounded for $t>0$ and $\left\{s_{n}\right\}$ is not $\left(A_{\mu}\right)$-convergent. But for $-1<\lambda<\mu, s_{n} \rightarrow O\left(A_{\lambda}\right)$ since $\sigma_{\lambda}(y)=\frac{\Gamma\left(\mu+1^{0}\right)}{\Gamma(\mu-\lambda) \Gamma(\lambda+1)} \frac{1}{y} \int_{0}^{y}(1-t / y)^{\mu-\lambda-1}(t / y)^{\lambda} \sin (\lambda+t) d t^{-}$ $\because=\frac{\Gamma(j+1)}{\Gamma(\mu-\lambda) \Gamma(\lambda+1)} \int_{0}^{1}(1-u)^{\mu-\lambda} u^{\lambda} \sin (\Gamma+y u) d u$

. $\rightarrow$ as $y+\infty$ by the Riemann-Lebesgue Theorem. Hence, Theorem 3.4 does not hold for $0=\mu-\lambda$. 

PROOF

We have

$j$

$$
\begin{aligned}
\int_{0}^{y} \frac{\sigma_{0}(t)}{1+t} d t & \equiv \int_{0}^{y} \frac{1}{(1+t)^{2}} \sum_{n=0}^{\infty} s_{n}\left(\frac{t}{1+t}\right)^{n} d t \\
& =\sum_{n=0}^{\infty} s_{n} \int_{0}^{y}\left(\frac{t}{1+t}\right)^{n} \frac{1}{(1+t)^{2}} d t \\
& =\sum_{n=0}^{\infty} \frac{s_{n}}{n+1}\left(\frac{y}{1+y}\right)^{n+1}
\end{aligned}
$$

3

The convergence of $\sum_{n=1}^{\infty} s_{n}\left(\frac{t}{1+t}\right)^{n}$ for $t>0$ implies its. absolute convergence for $t>0$, which justifies the term by term integration. This completes the proof.

- LEMMA 4.2

$$
\text { If } f(x) \text { is absolute lit continuous on }[0, T\} \text { : } 0 r \text { each } T>0
$$

and $\mathrm{f}^{\prime}(\dot{\mathrm{x}})>-\mathrm{M} / \mathrm{x}$ for $a l ? \mathrm{x}>0$, then $\mathrm{f}(\dot{x})$ is slow lu decreasing. PROOF ...

$$
\text { For }_{v} \varepsilon \geq 0 \text {, and } y>x>0
$$

$$
\begin{aligned}
f(y)-f(x) & =\int_{X}^{Y} f^{\prime}(t) d t \\
& >-M \int_{X}^{Y} \frac{d t}{t}
\end{aligned}
$$$$
=-M \ln y / x_{>}>-\varepsilon
$$

whenever $y / \dot{x}<\exp [\varepsilon / M]$. This completes the proof. 
4.3 A COUNTEREXAMPLE

THEOREM 4.1

There exists a sequence $\left\{\mathrm{s}_{\mathrm{n}}\right\}$ such that $\left\{\mathbf{s}_{\mathrm{n}}\right\}^{\circ}$ is (L)-cönvergent, and $\sigma_{0}(t)$ is slowly decreasing, but $\left\{\mathbf{s}_{\mathbf{n}}\right\}$ is not $\left(A_{\lambda}\right)$-convergent for any $\lambda>-1$.

PROOF

Let $\left\{s_{n}\right\}$ be the sequence such that

$$
(1-x) \sum_{n=0}^{\infty} s_{n} x^{n}=R\left\{(1-x)^{-i}\right\} \quad|x|<1 \text {. }
$$

The power series is convergent for $|x|<1$ and $\sigma_{0}(t)=R\left\{(1+t)^{i}\right\}$.

Now

$$
\begin{aligned}
t \sigma_{0}^{\prime}(t) & =-\sin (\log (1+t)) \frac{t}{1+t} \\
& >-1 \quad \text { for } t>0 .
\end{aligned}
$$

Hence, by Lemma $4.2, \sigma_{0}(t)$ is slowly decreasing.

clearly, $\left\{s_{n}\right\}$ is not $\left(A_{0}\right)$-convergent. Furthermore, by Theorems 1.2 and $3.1,\left\{s_{n}\right\}$ cannot be $\left(A_{\lambda}\right)$-convergent for any $\lambda>-1$.

However,

$$
\begin{aligned}
L(y) & =\frac{1}{\log (1+y)} \int_{0}^{y} \frac{\cos (\log (1+t))}{1+t} d t \\
& =\frac{\sin (\log (1+y))}{\log (1+y)} \rightarrow 0 \text { as } y \rightarrow \infty
\end{aligned}
$$

Hence, $s_{n} \rightarrow O(L)$.

This completes the proof. 
4.4 A TAUBERIAN THEOREM FOR (L) TO (A, SUMMABILITY

For the remainder of this chapter, we suppose that the slow decrease of a real-valued function, $f(t)$, is defined by the following.condition:

(4.4.1) given $\varepsilon>0$, there exist $x, \delta>0$ such that

$f(y)-f(x)>-\varepsilon$ whenever $y \geq x \geq x$ and

In $\ln y-\ln \ln x<\delta$.

THEOREM 4.2 .

If $\alpha>-1, s_{n} \rightarrow s(L)$, and condition (4.4.1) holas

with $f(t)=\sigma_{\alpha}(t)$, then. $s_{n} \rightarrow s\left(A_{\alpha}\right)$

4.5 METHODS OF SUMMABILITY BASED' ON POWER SERIES

Suppose that

$p_{n} \geq 0, q_{n} \geq 0, \sum_{v=n}^{\infty} p_{v}>0, \sum_{v=n}^{\infty} q_{v}>0$ for $n=0,1,2, \ldots$.

Let .

$p(x)=\sum_{n=0}^{\infty} p_{n} x^{n}, q_{0}(x)=\sum_{n=0}^{\infty} q_{n} x^{n}$ andraenote the radii

of convergence of these power series by $\rho_{p}$ and $\rho_{q}$

respectively.

We also write

$$
\begin{aligned}
& p_{s}(x)=\frac{1}{p(x)} \sum_{n=0}^{\infty} p_{n} s_{n} x^{n} \\
& q_{s}(x)=\frac{1}{q(x)} \sum_{n=0}^{\infty} q_{n} s_{n} x^{n} .
\end{aligned}
$$

If $\rho_{p}>0, \sum_{n=0}^{\infty} p_{n} s_{n} x^{n}$ is convergent in the

interval $\left(0, \rho_{p}\right)$ and $\lim _{x \rightarrow \rho_{p}} p_{s}(x)=8$, then we say that $\left\{s_{n}\right\}^{\prime}$ 
is summable to $s$ by the power series method (P) and write $s_{n} \rightarrow s(P)$. The method $(Q)$ is defined similarly.

We note that if $q_{n}=\frac{1}{n+1}$ for $n=0.1,2, \ldots$; then the method ' $(Q)$ is the logarithmic method $(L)$.

If $x(t)$ is a function of bounded variation on $[\dot{0}, 1]$, the associated normalized function $x^{*}(t)$ is defined as follows :

$$
x^{\star}(t)= \begin{cases}0 & t=0 \\ \frac{1}{2}[x(t+)+x(t-)] & -x(0) 0<t<1 \\ x(1)-x(0) & t=.1\end{cases}
$$

A segquence $\left\{\mu_{n}\right\}$ is said to be an $m$-sequence (moment sequence) if

$$
\mu_{n}=\int_{0}^{1} t^{n} d x(t) \quad(n=0,1,2, \ldots)
$$

where $x(t)$ is of bounded variation in $[0,1]$. If, in addition,

$$
\mu_{n} \geq \delta \int_{0}^{1} t^{n}\left|d x^{*}(t)\right|
$$

$(0<\delta \leq 1 ; n=N, N+1, \ldots)$ then we call $\left\{\mu_{n}\right\}$ an $\bar{m}$-sequence. We note that if $x(t)$ is of bounded variation in $[0,1]$, then $([15],-7 h e o r e m 8 a)$

1

$$
\int_{0}^{1} t^{n} d x(t)=\int_{0}^{1} t^{n} d x^{*^{-1}(t)}
$$

and :

$$
\begin{aligned}
& \text { ([16] p: } 14 \text { and [8] p. 335) } \\
& \int_{0}^{1} t^{n}|d x(t)| \geq \int_{0}^{1} t^{n}\left|d x^{*}(t)\right| .
\end{aligned}
$$


Hence an m-sequence $\left\{\mu_{n}\right\}$ such that

$$
\mu_{n}=\int_{0}^{1} t^{n} d x(t) \geq \delta \int_{0}^{1} t^{n}|d x(t)|
$$

where $0<\delta \leq 1$ and $n=N, N+1, \ldots$ is necessarily an $\bar{m}$-sequence.

LEMMA $4-3$

An m-sequence $\left\{\mu_{\Omega}\right\}$ which converges to a positive

: imit is an $\vec{m}$-sequence.

PROOF

Suppose, as we may, that

$$
\begin{aligned}
\mu_{n} & =\int_{0}^{1} t^{n} d x(t)=\int_{0}^{1} t^{n} d \alpha(t)-\int_{0}^{1} t^{n} d B(t) \\
& +u-v>0 \text { as } n+\infty:
\end{aligned}
$$

where $\alpha_{p}^{-g}(t), \beta(t)$ are non-decreasing, and bounded in $[0,1]$ and $u>v \geq 0$.

Then

$$
\begin{aligned}
\frac{\mu_{n}}{\int_{0}^{1} t^{n}|\dot{d} x(t)|} & =\frac{\int_{0}^{1} t^{n} d \alpha(t)-\int_{0}^{1} t^{n} d \beta(t)}{\int_{0}^{1} t^{n} d \alpha(t)+\int_{0}^{1} t^{n} d B(t)} \\
& \rightarrow \frac{u-v}{u+v}, \\
& \geq \frac{1}{2} \frac{u-v}{u+v}=\delta
\end{aligned}
$$


for sufficiently lairge $n$. The lemma follows by the observation immediately preceding the lemma.

We state two additional results required for proofs in the next section. Both are due to Borwein.

LEMMA 4.4

$\therefore \quad-I f \infty>\rho_{\mathrm{p}}>0$, ther, a necessary and sufficient condition for (P) to be regular. is that.

- (See [3], Theorem 1)

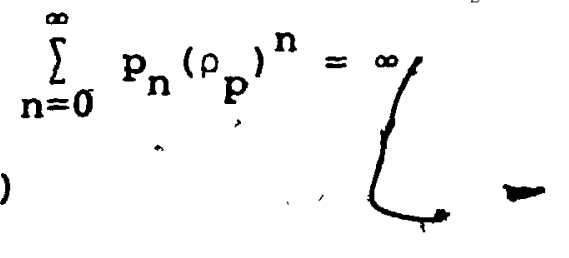

LEMMA 4.5

If $p_{n}=\mu_{n} q_{n}(n \geq N)$. where $\left\{\mu_{n}\right\}^{\prime i}$ is an $\bar{m}$-sequence,

if $\rho_{\mathrm{p}}=\rho_{\mathrm{q}}>0$ and $(\mathrm{P})$ is regular, then (Q') $\subseteq(\mathrm{P})$.

(See [ 3], Theorem A').

4.6 SंOME PRELIMIUARY LEMMAS

For the remainder of this chapter we set, for

$\alpha>-1$ and $w>0$,

(4.6.1) $J_{\alpha}(w)=\frac{1 \ldots}{\log (1+w)} \int_{0}^{w} \cdot(1+t)^{\alpha-1}\left[\log \frac{w(1+t)^{\alpha}}{t(1+w)}\right]^{\alpha} \sigma_{\alpha}(t) d t$ and"

$(4.6 .2) \quad v_{n i}^{i}=\frac{\varepsilon_{n}^{\alpha} \Gamma(\alpha+1)}{(n+1)^{\alpha}}$

LEMAN 4.7

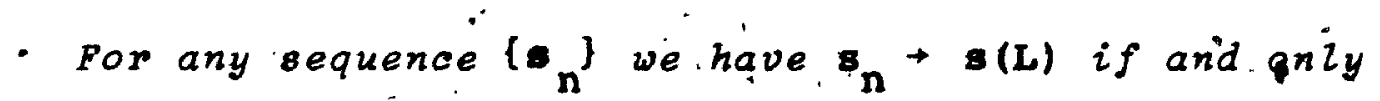
if $v_{n} s_{n} \rightarrow s(L)$. 
PROOF

$$
\text { suppose-s } s_{n} \rightarrow s(L) . \quad \text { set } q_{n}=\frac{1}{n+1} \text { and } p_{n}=v_{n} q_{n}
$$

for $n=0,1,2, \ldots$.

$\therefore$ Now since $v_{n}+1$, by Lemma 4.2 (and [7], Theorem 211) $\left\{v_{n} \cdot\right\}$ is an $\bar{m}$-sequence. Also, since $p_{n} \sim \frac{1}{n+1}$ as $n \rightarrow \infty$, we have by Lemma $\mathbf{4 . 4}$ that the method $(P)$. is regular, since $\sum_{n=0}^{\infty} \frac{\dot{i}^{\circ}}{n+1}=\infty$. Furthermore, $\rho_{p}=\rho_{q}=1$. Hence, by Lemma 4.5 (Q) $\subseteq(P)$. Since (Q) is (L) $\frac{1}{p(x)} \sum_{n=0}^{\infty} s_{n} \frac{v_{n}}{n+1} x^{n} \rightarrow$ as $x+1-\cdots$ Since $v_{n} \rightarrow 1$ and $(L)$ is regular

$$
\begin{aligned}
& \frac{-\operatorname{xop}(x)}{\log (1-x)}=-\frac{1}{\log (1-x)} \sum_{n=0}^{\infty} \frac{v_{n}}{n+1} x^{n+1} \rightarrow 1 \text { as } x \rightarrow 1^{-} \\
& \text {Therefore } \\
&-\frac{1}{\log (1-x)} \sum_{n=0}^{\infty} s_{n} \frac{v_{n}}{n+1} x^{n+1}= \cdot\left\{\frac{-x p(x)}{\log (1-x)}\right\}\left\{\frac{1}{p(x)} \sum_{n=0}^{\infty} s_{n} \frac{v_{n}}{n+1} x^{n}\right\} \\
& \rightarrow \text { s as } x \rightarrow 1^{-} .
\end{aligned}
$$

That is

$$
v_{n} s_{n} \rightarrow s(L)
$$

Now suppose $v_{n} \bar{s}_{n} \rightarrow s(L)$. Taking $h_{n}=1 / v_{n}$ and - $:$ considering the power series methods $(Q)$ and $(P)^{\prime} \cdot$ where $q_{n}=\frac{1}{n+1}$ and $p_{n}=h_{n} q_{n}$, we can show that $s_{n} \rightarrow g(L)$ by an argument. similar, to that above. 
$\downarrow 43$

This competes the proof.

LEMMA 4.8

If $\sigma_{\alpha}(t)$ is convergent for all $t>0$, then

$J_{0}(w)+s$ as $w+\infty$, if and only if $v_{n} s_{n} \rightarrow \mathbf{s}(L)$.

PROOF

$$
\begin{aligned}
& \text { Setting } u=\frac{t(1+w)}{w(1+t)} \text { in } J_{\alpha}(w) \text { gives } J_{\alpha}(w) \\
& =\frac{i}{\log (1+w)} \int_{0}^{w}(1+t)^{\alpha-f}(1+t)^{-\alpha-1} \sum_{n=0}^{\infty} \varepsilon_{n}^{\alpha} s_{n}\left(\frac{t}{1+t}\right)^{n}\left[\log \frac{w(1+t)}{t(1+w)}\right]^{\alpha} d t \\
& =\frac{1}{\log (1+w)} \int_{0}^{1}\left[\sum_{n=0}^{\infty} \varepsilon_{n}^{\alpha} s_{n}\left(\frac{w}{1+w}\right)^{n+1} u^{n}\right]\left[\log \frac{1}{u}\right]^{\alpha} d u \\
& =\frac{1}{\log (1+w)} \sum_{n=0}^{\infty} \varepsilon_{n}^{\alpha} s_{n}\left(\frac{w}{1+w}\right)^{n+1} \int_{0}^{1} u^{n}\left(\log \frac{1}{u}\right)^{\alpha} d u \\
& =\frac{\Gamma(\alpha+1)}{\log (1+w)} \sum_{n=0}^{\infty} \frac{\varepsilon_{n}^{\alpha}}{(n+1)^{\alpha+1}} s_{n}\left(\frac{w}{1+w}\right)^{n+1} \\
& =\frac{1}{\log (1+w)} \sum_{n=0}^{\infty} \frac{v_{n} s_{n}\left(\frac{w}{n+1}\right)^{n+1}}{1+w}
\end{aligned}
$$

Now, the convergence of the series defining $\sigma_{\alpha}(t)$ for $t>0$ implies its absolute convergence. This justifies the integration term by term and the proof is complete.

LEMMA 4.9

If $\alpha>-1, \sigma_{\alpha}(t)$ satisfies condition (4.4.1) and $s_{n} \rightarrow s(L)$ then $\sigma_{\alpha}(t)=0(1)(t \geq 0)$.

PROOF

Since it suffices to show that the result holds for $s=0$, we have by Lemma $4.7 v_{n} s_{n} \rightarrow 0(L)$, and hence by Lemma $4.8 J_{\alpha}(w) \rightarrow 0$ as $w \rightarrow \infty$. 
We show that the pre-conditions and conditions of Theorem 3.2 are satisfied with

$$
\begin{aligned}
& K(w, t)= \begin{cases}\frac{1}{\log (1+w)} & (1+t)^{\alpha-1}\left[\log \frac{w(1+t)}{t(1+w)}\right]^{\alpha} \quad 0<t<w \\
0, & \text { otherwise }\end{cases} \\
& \Phi(x)= \begin{cases}x / e^{e} & 0 \leq x<e^{e} \\
\ln \ln x & e^{e^{-}} \leq x\end{cases}
\end{aligned}
$$

and

$$
f(t)=\sigma_{\alpha}(t)
$$

Since

$$
\begin{aligned}
& \int_{0}^{\infty} K(w, t) d t=\frac{1}{\log (1+w)} \int_{0}^{w}(1+t)^{\alpha-1}\left[\log \frac{w(1+t)}{t(1+w)}\right]^{\alpha} d t \\
= & \frac{1}{\log (1+w)} \int_{0}^{w}(1+t)^{\alpha-1}\left[\log \frac{w(1+t)}{t(1+w)}\right]^{\alpha}(1+t)^{-\alpha-1} \sum_{n=0}^{\infty} \varepsilon_{n}^{\alpha}\left(\frac{t}{1+t}\right)^{n} d t
\end{aligned}
$$

we have, as in the proof of Lemma 4.8

$$
\begin{aligned}
\int_{0}^{\infty} K(w, t) d t & =\frac{1}{\log (1+w)} \sum_{n=0}^{\infty} \frac{v_{n}}{n+1}\left(\frac{w}{1+w}\right)^{n+1} \\
& \rightarrow 1 \text { as } w \rightarrow \infty
\end{aligned}
$$

since $v_{n} \rightarrow 1$ as $n \rightarrow \infty$ and the $(L)$ method is regular. This establishes the preconditions of Theorem 3.2 .

Conditions $(3.2 .1),(3.2 .2)$, and $(3.2 .3)$ clearly

hold.

Furthermost, since $k(w, t)=0$ whenever $t \geq w,(3.2 .5)$

- holds. It remains to show that $(3.2 .4)$ is satisfied, since (3.2.6) holds by the hypothesis, $s_{n} \rightarrow O(L)$. 
For $\alpha \geq 0$ and $x \geq 1$, we have.

$$
\begin{aligned}
& \log (1+w) \int_{0}^{x} K(w, t) d t \\
= & \int_{0}^{x}(1+t)^{\alpha-1}\left[\log \frac{w(1+t)}{t(1+w)}\right]^{\alpha} d t \\
\leq & \int_{0}^{x}(1+t)^{\alpha-1}\left[\log \frac{\left.\frac{1+t}{t}\right]^{\alpha} d t}{d}\right. \\
= & \left.\int_{0}^{1}+\int_{1}^{x}\right]^{(1+t)^{\alpha-1}}\left(\log \frac{1+t}{t}\right)^{\alpha} d t \\
= & I_{1}+I_{2} \cdot
\end{aligned}
$$$$
\text { Setting } u=1 / t \text { in } I_{1} \text { gives }
$$$$
\begin{aligned}
I_{1} & =\int_{1}^{\infty}(1+1 / u)^{\alpha-1}[\log (1+u)]^{\alpha} \frac{d u}{u^{2}} \\
& =0(1)=0(\log (1+w))
\end{aligned}
$$$$
I_{2}=\int_{1}^{x}(1+t)^{\alpha-1}[\log (1+1 / t)]^{\alpha} d t
$$

J.

$$
=0(1) \int_{1}^{x}(1+t)^{-1} d t
$$$$
=0(1) \log (1+x)+0(1) \text {. }
$$

Therefore

$$
\begin{aligned}
& \text { Therefore } \frac{1}{\log (1+w)}\left(I_{1}+I_{2}\right) \\
& =0(1)+0(1) \frac{\log (1+x)}{\log (1+w)} \\
& =0(1) \text { as } w>x+\infty \text { and } \ln \ln w-\ln \ln x+\infty
\end{aligned}
$$


Hence, condition (3.2.4) holds fot $\alpha \geq 0$.

In the case $-1<\alpha<0$, we first observe that for $0<t<w$ we have

$$
\log \frac{w(1+t)}{t(1+w)}>\frac{w-t}{w(1+t)}
$$

since, for $y>1$

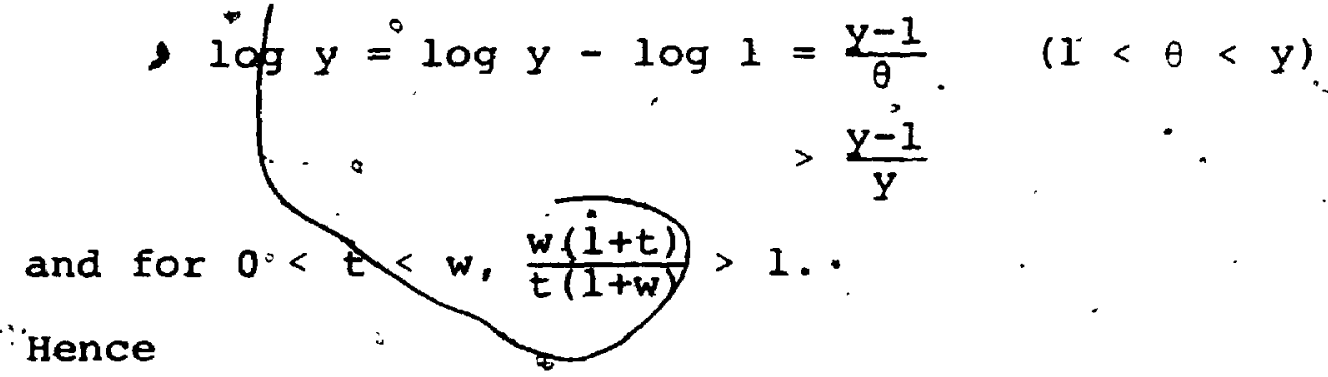

$$
\begin{aligned}
& \frac{1}{\log (1+w)} \int_{0}^{x}(1+t)^{\alpha-1}\left[\log \frac{w(1+t)}{t(1+w)}\right]^{\alpha} d t \\
\leq & \frac{1}{\log (1+w)} \int_{0}^{x} \cdot(1+t)^{\alpha-1}\left[\frac{w-t}{w(1+t)}\right]^{\alpha} d t \\
= & \frac{1}{\log (1+w)} \int_{0}^{x} \cdot(1-t / w)^{\alpha} \frac{d t}{1+t^{2}-\cdots} \\
\leq & \frac{(1-x / w)^{\alpha}}{\log (1+w)} \int_{0}^{x} \frac{d t}{1+t} .
\end{aligned}
$$

D.

$$
=0(1) \frac{\log (1+x)}{\log (1+w)}=0(1) \text { as }
$$

$w>x+\infty$ and $\ln \ln w-\ln \ln x \rightarrow \infty$, since the latter implies $\frac{\ln x}{\ln w} \rightarrow 0$ and $x / w \rightarrow 0$. This establishes condition $(3.2 .4)$ for $\alpha>-1$ and rence the lemma.

We now give a direct proof of Theorem 1.4. 
THEOREM 4.3

$$
\left(A_{-\alpha}\right) \subseteq \text { (L) for } \alpha>-1
$$

PROOF

The thearem is an immediate consequence of

Lemunas $4.7,4.8$ and 3.1 with

$R(w, t)=\left\{\begin{array}{cc}\frac{1}{\log (1+w)}(1+t)^{-\alpha-1}\left[\log \frac{w(1+t)}{t(1+w)}\right]^{\alpha} 0<t<w \\ 0 \cdot\end{array}\right.$

$$
\Phi(x)= \begin{cases}x / e^{e} & 0 \leq x<e^{e} \\ \ln \ln x & e^{e} \leq x\end{cases}
$$

since condition $(3.2 .4)$ is established as in Lemma 4.9 and the other conditons are clearly satisfied.

IEMMA 4.30

$\lambda$

$$
\begin{gathered}
\text { If } \gamma>1 \text {, and } \alpha>^{\prime}-1 \text { then } \\
I(x)=\int_{0}^{x}(1+t)^{\alpha-1}\left\{\left[\log \frac{x^{\gamma}(1+t)}{t\left(1+x^{\gamma}\right)}\right]^{\alpha}-\left[\log \frac{x(1+t)}{t(1+x)}\right]^{\alpha}\right\} d t=0(1) .
\end{gathered}
$$

PROOF

$$
\begin{aligned}
& \text { Süppose } \alpha \geq 1 \text { : Then if } x \geq 1 \\
& |I(x)|==^{\circ} I(x) \leq \alpha \log \frac{x^{\gamma}(1+x)}{x\left(1+x^{\gamma}\right)} \int_{0}^{x}(1+t)^{\alpha-1}\left[\log \frac{x^{\gamma}(1+t)}{-t\left(1+x^{\gamma}\right)}\right]^{\alpha-1} d t
\end{aligned}
$$

$$
\leq \alpha \log \frac{x^{\gamma}(1+x)}{\left(1+x^{\gamma}\right) x}\left(\int_{0}^{1}+\int_{1}^{x}(1+t)^{\alpha-1}\left(\log \frac{1+t}{t}\right)^{\alpha-1} d t\right.
$$


;Now,

$\therefore$

$$
\begin{aligned}
\int_{0}^{1}(1+t)^{\alpha-1}\left(\log \frac{I+t}{t}\right)^{\alpha-1} d t & =\int_{1}^{\infty}(1+i / u)^{\alpha-1}[\log (1+u)]^{\alpha-1} \frac{d u}{u^{2}} \sum^{\infty} \\
\cdots & <\infty
\end{aligned}
$$

Hence,

$$
\alpha \log \frac{x^{\gamma}(1+x)}{x\left(-1+x^{\gamma}\right)} \int_{0}^{i}(1+t)^{\alpha-1}\left(\log \frac{i+t}{t}\right)^{\alpha-1} d t=0(1) \text {. }
$$

Also, .

$$
\begin{aligned}
& \quad a \log \frac{x^{\gamma}(1+x)}{x\left(1+x^{\gamma}\right)} \int_{1}^{x}(1+t)^{\alpha-1}\left(\log \frac{1+t}{t}\right)^{\alpha-1} d t \\
& \quad=0(1) \log \frac{x^{\gamma}(1+x)}{x\left(1+x^{\gamma}\right)} \cdot \int_{1}^{x} d t \\
& \quad \quad \leq 0(1) \times \log \frac{1+x}{x}=0(1) .
\end{aligned}
$$

Suppose $0<a<1$. As established in the proof of

Lemma $4.9^{\circ}$

$$
\log \frac{x(1+t)}{t(1+x)}>\frac{x-t}{x(1+t)} \quad(0<t<x) \text {. }
$$

we' have

$$
\begin{aligned}
& \mid \frac{O}{I(x) \mid}=I(x) \leq \alpha \log \frac{x^{\gamma}(1+x)}{x\left(1+x^{\gamma}\right)} \int_{0}^{x}(1+t)^{\alpha-1}\left[\log \frac{x(1+t)}{t(1+x)}\right]^{\alpha-1} d t \\
& \leqq \alpha \frac{M}{x} \cdot \int_{0}^{x}(1+t)^{\alpha-1}\left[\frac{x-f}{x(1+t)}\right]^{\alpha-1} d t \\
& \text { since }\left|x \log \frac{x^{Y}(1+x)}{x\left(1+x^{Y}\right)}\right| \leqslant \text { Therefore } \\
& |I(x)| \leq \frac{\alpha M}{x^{\alpha}} \int_{0}^{x}(x-t)^{\alpha-1} d t=M
\end{aligned}
$$


49

- Suppose $-1<\alpha<0$. Then

.

$$
\begin{aligned}
\left|I\left(x^{\alpha}\right)\right| & =-I(x)=\int_{0}^{x / 2}(1+t)^{\alpha-1}\left\{\left\{\left[\log \frac{x(1+t)}{t(1+x)}\right]^{\alpha}-\left[\log \frac{x^{\gamma}(1+t)}{t\left(1+x^{\gamma}\right)}\right]^{\alpha}\right\} d t\right. \\
& +\int_{x / 2}^{x}(1+t)^{\alpha-1}\left\{\left[\log \frac{x(1+t)}{t(1+x)}\right]^{\alpha}-\left[\log \frac{x^{\gamma}(1+t)}{t\left(1+x^{\gamma}\right)}\right]^{\alpha}\right\} d t \\
& =I_{1} \cdot(x)+I_{2}(x) .
\end{aligned}
$$

$$
\begin{aligned}
& 0 \leq I_{1}(x) \leq \alpha \log \frac{x\left(x+x^{\gamma}\right)}{x^{Y}(1+x)} \int_{0}^{x / 2}(1+t)^{\alpha-1}\left[\log \frac{x(1+t)}{t(1+x)}\right]^{\alpha-1} d t \\
& \leq \frac{-\alpha M}{x} \int_{0}^{x / 2}(1+t)^{\alpha-1}\left[\frac{x-t}{x(1+t)}\right]^{\alpha-1} d t \\
& \text { since }\left|x \log \left(\frac{x}{1+x} \cdot \frac{1+x^{\gamma}}{x^{\gamma}}\right)\right| \leq M^{\prime} \text { Therefore } \\
& I_{1}(x) \leq-\frac{\alpha M}{x^{\alpha}} \int_{0}^{x / 2}(x-t)^{\alpha-1} d t . \\
& =M\left[\left(\frac{1}{2}\right)^{\alpha}-01\right] \text {. } \\
& \text { Finally, } \\
& 0 \leq I_{2}(x) \cdot \leq \int_{x / 2}^{x}(1+t)^{\alpha-1}\left[\log \frac{x(1+t)}{t(1+x)}\right]^{\alpha} d t \\
& \leq \int_{x / 2}^{x}(1+t)^{\alpha-1}\left[\frac{x-t}{x(1+t)}\right]^{\alpha} d t \\
& =\frac{1}{\alpha} \int_{x / 2}^{x}(x-t)^{\alpha} \frac{d t}{1+t} \text {. }
\end{aligned}
$$$$
3
$$ 
Now, since $1+t>x / 2$

$$
\begin{aligned}
I_{2}(x) & \leq \frac{2}{x^{\alpha+1}} \int_{x / 2}^{x}(x-t)^{\alpha} d t \\
& =\frac{2}{x^{\alpha+1}} \frac{1}{\alpha+1}\left(\frac{x}{2}\right)^{\alpha+1}=\frac{1}{(\alpha+1) 2^{\alpha}}
\end{aligned}
$$

Hence, $\dot{I}(x)=0(1)$ in this case.

Finally, since the case $\alpha=0$ is trivial the lemma is established.

LEMMA 4.11

$$
\begin{aligned}
& \text { If } \gamma>1 \text {, and } \alpha>-1 \text {, then } \\
& \int_{x}^{x^{\gamma}}(1+t)^{\alpha-1} \cdot\left[\log \frac{x^{\gamma}(1+t)}{t\left(1+x^{\gamma}\right)}\right]^{\alpha} d t .
\end{aligned}
$$

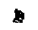

$$
\begin{aligned}
& =(\gamma-1) \log (1+x)+0(\log (1+x)) .
\end{aligned}
$$

PROOF

If $\sigma_{\alpha}(t)=1$ for $t>0$, then by Lemmas $4.7,4.8$

and Theorem $1.4 J_{\alpha}(x)=1+0(1)$ as $x \rightarrow . \infty$.

Now, by Lemma $4: 10$

$$
\begin{aligned}
& \int_{x}^{x^{\gamma}}(1+t)^{\alpha-1} \log \left[\frac{x^{\gamma}(1+t)}{t\left(1+x^{\gamma}\right)}\right]^{\alpha} d t . \\
= & \int_{0}^{x^{\gamma}}-\int_{0}^{x}(1+t)^{\alpha-1}\left[\log \frac{x^{\gamma}(1+t)}{t\left(1+x^{\gamma}\right)}\right]^{\alpha} d t \\
= & \log \left(1+x^{\gamma}\right)+0\left(\log \left(1+x^{\gamma}\right)\right)-\log (1+x)+o(\log (1+x)) \\
= & 0(1) \\
= & (\gamma-1) \log (1+x)+0(\log (1+x))
\end{aligned}
$$

This establishes the lemma. 


\subsection{PROOF OF THEOREM 4.2}

By $(4.4 .1)$, for $\varepsilon>0$, there exist an $x, \delta>0$

such that

$(4.7 .1) \quad \sigma_{\alpha}(y)-\sigma_{\alpha}(x)>-\varepsilon$ whenever $y>x>x$ and $\ln \ln y-\ln \ln x<\delta$. That is, writing $\delta=\ln \gamma$, $\sigma_{\alpha}(x)-\varepsilon<\sigma_{\alpha}(y)$ whenever $x<x<y<x^{\gamma}$.

We may, without loss of generality, suppose that $s_{n} \rightarrow O(L)$. Hence, by Lemma 4.7, $v_{n} s_{n} \rightarrow O(L)$. That is, $J_{\alpha}(x)+0$ as $x \rightarrow \infty$. Therefore if $x>x$

(4.7.2) $\int_{x}^{x_{\gamma}^{\gamma}}(1+t)^{\alpha-1}\left[\log \frac{x^{\gamma}(1+t)}{t\left(1+x^{\gamma}\right)}\right]^{\alpha}\left[\sigma_{\alpha}(x)-\varepsilon\right] d t$
)$\leq \int_{x}^{x}(1+t)^{\alpha-1}\left[\log \frac{x^{\gamma}(1+t)}{t\left(1+x^{\gamma}\right)}\right]^{\alpha} \sigma_{\alpha}(t) d t$

$$
\begin{aligned}
& =\int_{0}^{x^{\gamma}}-\int_{0}^{x}\left[(1+t)^{\alpha-1}\left[\log \frac{x^{\gamma}(1+t)}{t\left(1+x^{\gamma}\right)}\right]^{\alpha} \sigma_{\alpha}(t) d t\right. \\
& =\log \left(1+x^{\gamma}\right) J_{\alpha}\left(x^{\gamma}\right)-\log (1+x) J_{\alpha}(x)+0(1)
\end{aligned}
$$

by Lemma 4.10. Now, $J_{\alpha}^{\prime}(x) \rightarrow 0$.

Therefore

$$
\begin{aligned}
&(4.7 .3) \int_{x}^{x^{\gamma}}(1+t)^{\alpha-1} i\left[\log \frac{x^{\gamma}(1+t)}{t\left(1+x^{\gamma}\right)}\right]^{\alpha} \cdot \sigma_{\alpha}(t) d t \\
&=o\left(\log \left(1+x^{\gamma}\right)\right)+o(\log (1+x)) \\
&=o(\log (1+x)) . \\
& \text { But, by Lemma 4.11. }
\end{aligned}
$$




$$
\begin{aligned}
& \left(\sigma_{\alpha}(x)-\varepsilon\right) \int_{x}^{x^{\gamma}}(1+t)^{\alpha-1}\left[\log \frac{x^{\gamma}(1+t)}{t\left(1+x^{\gamma}\right)}\right]^{\alpha} d t \\
& \risingdotseq\left(\sigma_{\alpha}(x)-\varepsilon\right)\left[(\gamma-1)-\log (1+x)_{\infty}+\circ(\log (1+x))\right] .
\end{aligned}
$$

Therefore.

$$
\left(\sigma_{\alpha}(x)-\varepsilon\right)[(\gamma-1) \log (1+x)+o(\log (1+x))] \leq o(\log (1+x)) \text {. }
$$

That is,

$$
\sigma_{\alpha}(x)-\varepsilon \leq \frac{o(1)}{(\gamma-1)+o(1)}
$$

and hence

$$
\text { (4.7. 4) } \lim _{x \rightarrow \infty} \sup \sigma_{\alpha}(x) \leq \varepsilon
$$

Rewriting $(4.7 .1)$ we have

$\sigma_{\alpha}(x)<\sigma_{\alpha}(y)+\varepsilon$ whenever $x<y^{1 / \gamma}<x<y$.

Furthermore, by $(\overline{4} .7 .3)$

(replacing $x$ by $x^{1 / Y Y}$ ) we have

$$
\begin{aligned}
\circ\left(\log _{a}(1+y)\right) & =\int_{y^{1 / \gamma}(1+t)^{\alpha-1}\left[\log \frac{y(1+t)}{t(1+y)}\right]^{\alpha} \cdot \sigma_{\alpha}(t) d t}^{y} \\
& \leq \int^{y}(1+t)^{\alpha-1}\left[\log \frac{y(1+t)}{t(1+y)}\right]^{\alpha}\left(\sigma_{\alpha}(y)+\varepsilon\right) d t \\
& =\left(\sigma_{\alpha}(y)+\varepsilon\right)\{(1-1 / \gamma) \log (1+y)+o(\log (1+y)\} .
\end{aligned}
$$

Hence,

$$
\frac{o(1)}{(1-1 / \gamma)+o(1)} \leq \dot{\sigma}_{\alpha}(y)+\varepsilon
$$

- and therefore

$$
\text { (4.7.5) }-\varepsilon \leq \lim _{y \rightarrow \infty} \inf , \sigma_{\alpha}(y)
$$


Combining $(4.7 .4)$ and $(4.7 .5)$ complewes the proof of the theorem.

4.8 A CONDITION ON \{na $\left.{ }_{\mathrm{n}}\right\}$ WHICH IMPLIES RELATION (4.4.1)

We say $\left\{s_{n}\right\}=O_{L}(f(y))\left(A_{\lambda}\right)$ if there exist a $Y_{0}$ and an $H>0$ such that $f(y)$ is defined and positive for $y y_{0}$ and $\sigma_{\lambda}(y):-M f(y)$ for $y \geq y_{0}$.

We also set

$$
t_{\lambda}(y)^{-}=(1+y)^{-\lambda-1} \sum_{n=0}^{\infty} \varepsilon_{n}^{\lambda} n a_{n}\left(\frac{y}{1+y}\right)^{n}
$$

for $y>0$

It is easily shown that

$y \frac{d}{d y} \sigma_{\lambda}(y)=(\lambda+1)\left\{\sigma_{\lambda+1}(y)-\sigma_{\lambda}(y)\right\}=t_{\lambda}(y)$.

Furthermore, as in the proof of Lemma $4.2, \sigma_{\lambda}(y)$ satisfies condition $(4.4,1)$ if $\sigma_{\lambda}^{\prime}(y)>-M / y^{l n}(y$ for sufficiently large $y$.

Hence, $\sigma_{\lambda}(y)$ satisfies, $(4: 4.1)$-if

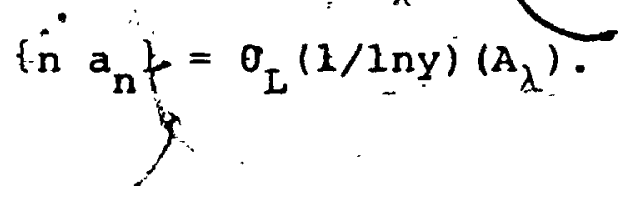




\section{CHAPTER 5 \\ A TAUBERIAN THEOREM FOR \\ ABELIAN SUMMABILITY METHODS}

5.1 INTRODUCTION

In this chapter we assume, as in section 1.5 , that

$$
0 \leq \lambda_{1}<\lambda_{2}<\cdots ; \lambda_{n} \rightarrow \infty
$$

and prove a general tauberian theorem for the abelian summability method $(A, \lambda)$. .

THEOREM 5.1

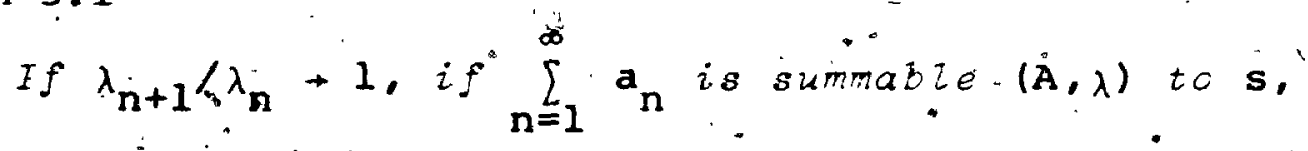

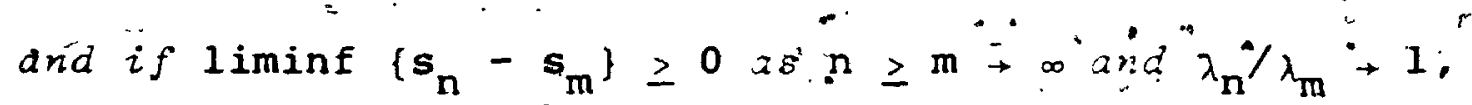
then $\mathbf{s}^{*} \rightarrow$

- When $\lambda_{n}=\bar{n}$, then $(A, \lambda)$ method is's equivalent to the ordinary Abel summability method $A_{0}$, and the result:in this case is well-known (see, for example, [ 7 ], Theorem 106): The only other casé which appears to be known is $\lambda_{n}=\log n$. The result in this case was established by kwee [10] by a method fundamentally different from and more complicated than ours.

\subsection{A'PRELIMINARY LEMMA}

We use the following known result (see [7], p. 164, Theorem 105t: 
$\therefore$

0

LEMMA 5.1

If $\alpha$ is a function of bounded variation. avery

$$
\text { interval [0,T]: if } \int_{0}^{\infty} e^{-y t} d_{\alpha}(t) \text { is convergent for } y>0
$$

- and tends to s as $y, 0+$, and if 1 imine $\{\alpha(y)-\alpha(x)\} \geq 0$ as $y \geq x+\infty$ and $y / x^{\circ} \rightarrow 1$, then ${ }^{-} \alpha f(t) \rightarrow s$ as $t+\infty$.

5.3 PROOF, OF THEOREM 5.1

set

$$
a(t)=\sum_{\lambda_{\mathrm{r}}<t} a_{n}
$$

Then for $y>0$, we have

$$
\int_{0}^{\infty} e^{-y t} d \alpha(t)=\sum_{n=1}^{\infty} a_{n} e^{-y \lambda_{n}}
$$

and, by hypothesis, the series converges and its sum tends to s as $y \rightarrow 0+$.

Assign $\varepsilon>0$. Then there exist positive numbers $M, \delta$ such that $s_{n}-s_{m}>-\varepsilon$ whenever $n \geq m \geq M$ and $\lambda_{n} / \lambda_{m} \leq 1+2 \delta . \quad$ Choose an. integer $N$ such that $\lambda_{N}>\lambda_{M+1}$ and, for $m+1 \geq N$,

$$
\frac{\lambda_{\mathrm{m}+1}}{\lambda_{\mathrm{m}}} \leq \frac{1+2 \delta}{1+\delta}
$$

Let $y \geq x \geq \lambda_{N}$ and $y / x \leq 1+\delta$

Then there exist integers $n, m$ such that

$$
\begin{aligned}
& \lambda_{n+1} \geq Y>\lambda_{n} \text { and } \lambda_{m+1} \geq x>\lambda_{m} . \text { Hence } n \geq m \geq M \text { and } \\
&{ }_{\&}^{\lambda_{\mathrm{m}}}<\frac{y}{x}-\frac{\lambda_{m+1}}{\lambda_{m}} \leq(1+\delta) \frac{1+2 \delta}{1+\delta}=1+2 \delta ;
\end{aligned}
$$


56

and therefore

$$
\begin{aligned}
\alpha(y)-\alpha(x) & =\alpha\left(\lambda_{n+1}\right)-\alpha\left(\lambda_{m+1}\right) \\
& =s_{n}-s_{m}>-\varepsilon .
\end{aligned}
$$

$\therefore$ Consequently liming $\{\alpha(y)-\alpha(x)\} \geq 0$ as

$$
y, \geq x \rightarrow \infty \text { and } y / x \rightarrow 1 \text {; and so; by Lemma } 5.1, s_{n} \rightarrow s \text {. }
$$

This completes the proof. 
REFERENCES

[1] A. Amir.

"On a converse of Abel's theorem",

Proc American Math: Soc.;

3 , (1952), 244-256.

[2] D. Borwein,

W"On a scale of Abel-type summability methods", Proc. Cambridge Phil. Soc.,

53 , (1957), 318-322.

[3] D. Borwein,

"On methods of summability based on power series", Proc. Royal Soc. Edinburgh, 64, (1957), 342-349.

[4] D. Borwein,

"A logarithmic method of summability",

Journai Londox Math. Soc.,

$33,(1958), \div 212-220$.

[5] D. Borwein,

"Theorems on some methods of summability" ,

Quarterly Journal Math. (2).

9. (1958), 310-316.

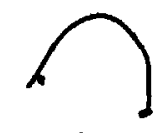


[6] D. Borwein,

"On moment constant methods of summability", Journal London Math. Soc.", 35, (1960), 71-77.

[7] G. H. Hardy,

Divergent Series

oxford

(1949).

[8] E. W. Hobson,

The Theory of Functions of a Real Variable, I, Cambridge (1927).

[9] E. Kogbetliantz.

Sommation des géries integrales,

- Mémor. Sci. Math. No. 51 .

[10] B. Kwee.

"Some theorems on the $(A, \log n)$ method of sumation".

7 Journal Lọndon Math. Soc., (2), $1(1969), 323-330$.

[11] R. D. Lord.

-The Abel, Borel, and Cesaro methods of sumation",

Proc. London Math. Soc.,

$(2), 38(1934,5), 241-256$. 
[12] R. J. Phillips,

Scales of Logarithmic Summability,

$\mathrm{Ph} . \mathrm{D}$. Thesis

- University of Western Ontario, (1968).

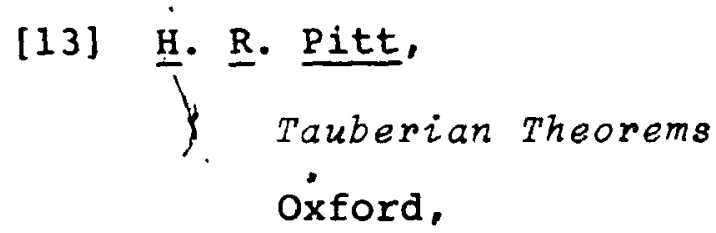

(1958).

[14] S. J. ‥ Rizvi,

Abel-Type Summability

Ph.D. Thesis,

University of Western Ontario.

(19̣69)

[15] T. Vijayaraghavan,

"A tauberian theorem",

Journal London Math. Soc.,

1 (1926), 113-120.

[16] D. V: Widder,

The Laplace Transform

Princeton,

(1946)

[17] D. . V. Widder,

An Introduction to Transform Theory,

Academic Press,

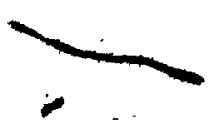

(1971) . 
$\because 60$

[18] N. Wiener,

"Tauberian theorems",

Annals of Mati்.,

(2), $33(1932) ; 1-100$.

[19]. N. Wiener,

The Fourier.Integral

Cambridge University Press,

$\therefore$ (1933). 